\title{
A regression-based damage detection method for structures subjected to changing environmental and operational conditions
}

\author{
William Soo Lon Wah ${ }^{1 *}$, Yung-Tsang Chen ${ }^{2}$ and John S Owen ${ }^{3}$ \\ ${ }^{1}$ The Waikato Institute of Technology, New Zealand \\ ${ }^{2}$ The University of Nottingham Ningbo China \\ ${ }^{3}$ Faculty of Engineering, The University of Nottingham
}

\begin{abstract}
Damage detection of civil engineering structures during the past decade has focused on eliminating the effects of the changing environmental and operational conditions, from the effects of damage. In the literature, a regression analysis has been adopted to construct a model between the vibration properties of structures, and the environmental and operational parameters to represent the undamaged state of the structures, for damage detection. However, using the environmental and operational parameters in the analysis has several limitations. For example, these parameters are not always available which may affect the performances of the damage detection methods. Regression between the vibration properties only has also been proposed in the literature where multivariate statistical tools have been adopted to extract the relationships among the properties. However, these methods have the problem that it is more difficult to detect damage in the multivariate situations and a regression target is usually needed, which is difficult to determine. Therefore, a damage detection method which uses the simple regression analysis, is developed in this paper. The vibration properties of structures are used as both the independent and dependent variables in the developed method. This has the advantages that the environmental and operational conditions are not needed and the multivariate statistical tools are not required for data processing. The developed method is applied to a beam structure model and the Z24 Bridge, in Switzerland, and the results obtained demonstrate that the method can successfully classify between undamaged and damaged states. The traditional regression analysis method is also applied to the two structures and it was found that better results are obtained using the method developed in this paper.
\end{abstract}

Keywords: Damage detection, regression analysis, natural frequencies, environmental and operational conditions, temperature conditions, outlier analysis

\section{Introduction}

Damage detection of civil engineering structures during the past decade has focused on eliminating/separating ${ }_{20}$ the effects of the changing environmental and operational 5 conditions (e.g. temperature) the structures face, from the effects of damage. This is because the changing environmental and operational conditions affect the vibration properties (e.g. natural frequencies) of structures which ${ }_{25}$ are commonly analysed to detect the presence of damage.

10 False alerts may occur if these conditions are not taken into account [1, 2].

In the literature, it was found that the vibration properties of structures are affected by multiple environmental ${ }_{30}$ and operational conditions (e.g. temperature [3, 4, 5, 6, 7,

15 8, 9, traffic loading [8, 10, 11, 12, wind loading [11, 13. and humidity [14]). For example, it was reported that the

Email address: William.soolonwah@wintec.ac.nz (William Soo Lon $\mathrm{Wah}^{1 *}$ ) natural frequencies of the Z24 Bridge, in Switzerland fluctuated due to the changing temperature conditions, and a nonlinear relationship existed between the frequencies and the temperature [3. Jin et al. 6] also reported similar relationship between the natural frequencies of the Meriden Bridge and the temperature condition. It was also found that traffic loading causes variations in the natural frequencies of structures. For example, the natural frequencies of the Tamar Bridge [11] and the Forth Road Bridge [12, in the United Kingdom, and the Sutong Cable-Stayed Bridge [8], in China were found to vary due to the traffic loading on the bridges. Some studies also reported that wind loading [11, 13] and humidity [14] altered the natural frequencies of civil engineering structures. Therefore, all these effects need to be considered while developing damage detection methods.

To take these environmental and operational conditions into account for damage detection, researchers have 35 adopted several approaches. These approaches use the fact that damage, which is a local phenomenon, affects the vi- 
bration properties locally. In other words, the modes of vibration are affected by structural damage and each mode 95 of vibration is affected to a different degree [15. There-

40 fore, damage can be detected in two ways; the first is by analysing the changes in vibration properties due to structural damage, and the second is by analysing the difference in changes among the modes of vibration which is created ${ }_{100}$ by damage.

One popular approach proposed for damage detection which analyses the changes in vibration properties, is the regression analysis approach. In this approach, a regression analysis is adopted to create a model between the 105 vibration properties of structures, and the environmental

50 and operational parameters, to represent the normal (undamaged) conditions of the structures [3, 16, 17, 18, 19, $20,21,22]$. When damage occurs, the vibration properties will be affected and for the same environmental and opera-110 tional conditions, the structures will have two different sets damaged and damaged). Therefore, new measurements can be compared to the model created and a large deviation from the model can then be attributed to an abnormal ${ }_{115}$ condition. In this paper, the abnormal condition is due to damage of structural components. In the regression analysis, the independent variable(s) are the environmental and operational parameters, while the dependent variable(s) are the vibration properties of structures. This regression 120 analysis approach proposed in the literature will be re-

65 ferred to as the traditional regression analysis approach throughout the paper.

Although conceptually simple, this approach carries several limitations. For example, this traditional regres- 125 sion approach relies on the availability of the measure-

70 ments of the environmental and operational conditions 22]. These measurements are not always available, which may affect the performances of the damage detection methods. Moreover, the optimal locations to place the sensors 130 to record the environmental and operational conditions

75 may be difficult to determine or to reach 223. Also, once the regression models have been created, the sensors need to stay at their original locations. Failure in any of the sensors may affect the models and hence the damage de-135 tection methods. Large-scale structures such as long-span so bridges and tall buildings are also subjected to different environmental and operational conditions at different locations 2. For example, gradient temperature conditions along the structures may exist which makes the analysisi40 more difficult and requires more sensors to be placed on the

85 structures. Generally, a large number of sensors are used for monitoring, however, only a few of the sensors are suitable to construct the regression model. For example, for the Ting Kau Bridge, in Hong Kong, Ko et al. 24] used ${ }_{145}$ 20 sensors out of 83 sensors installed permanently on the 90 structure to construct a natural frequencies-temperature model of the bridge. Due to all these aforementioned problems, it is desired to develop a damage detection method that does not require the environmental and operational ${ }_{150}$ parameters, for analysis.

To eliminate the use of the environmental and operational parameters, researchers have proposed to analyse the relationships among the vibration properties only using multivariate statistical tools (e.g. Principal Component Analysis and Cointegration) [9, 23, 25, 26, 27, 28, 29]. This approach is similar to the regression analysis approach, however, instead of analysing the relationships between the vibration properties and the environmental and operational parameters, this approach analyses the relationships among the vibration properties only. When damage occurs, each mode of vibration will be affected to a different degree. This will change the relationships among the vibration properties which can then be analysed using the multivariate statistical tools. For example, Cointegration was adopted to find a linear combination of the vibration properties (or other damage sensitive features) to construct a stationary model error to represent the undamaged state of the structures [25, 26, 30. When damage occurs, the model error will become nonstationary and damage can be alerted. Principal Component Analysis was also adopted to extract new features from the vibration properties data set that are sensitive to damage but less sensitive to the changing environments $9,23,26,27,28$. Selected features can then be analysed to detect damage.

However, using the multivariate statistical tools to analyse the relationships among the vibration properties has the problem that it is more difficult to detect damage in a data set containing multiple variables than the univariate case as the damage effect may be hidden in the data mass 31. Also, some methods require the use of a regression target during analysis. To reduce false alerts, the least affected and most affected modes of vibration need to be compared to each other in these methods to maximise the model error, hence, one of these modes need to be the regression target. However, it is difficult to choose this regression target as different damage scenarios (different damage locations and extent) affect the vibration properties differently. To limit this problem, several multivariate models were analysed using Cointegration in the literature where a model was created for each mode of vibration [30. Moreover, this multivariate statistical tools approach has the problem that the new features extracted are assumed to represent the damage effect and the environmental and operational effects, separately. In reality, the features may represent a combination of both effects. Furthermore, if too many or too few features are extracted and analysed, the effects of the environments may be underestimated or overestimated, which may lead to false alerts. Due to all these aforementioned problems, it is ideal to develop a method that does not require complicated multivariate statistical tools to analyse the relationships among the vibration properties.

Therefore, a damage detection method which analyses the changes between the natural frequencies of different modes of vibration, is developed in this paper. The method adopts the simple regression analysis approach for 
data processing. However, compared to the traditional re-205 gression analysis approach, the method developed in this paper uses the natural frequencies of structures as both the independent and dependent variables. This removes the need to use the environmental and operational parameters in the analysis. Using the regression analysis method ${ }_{210}$ also removes the need to extract new features and also does not require the analysis of multiple modes of vibration in a single data set. This reduces the occurrence of false alerts. Moreover, to increase the sensitivity to damage, the method analyses the relationship between the least af-215 fected and the most affected modes of vibration.

To test the method developed in this paper, a numerical beam model and the Z24 Bridge, in Switzerland are analysed. Since the method developed uses the regression approach, it is also compared with the traditional regres-220 sion analysis method. The results obtained demonstrate that the cases are well classified (between damaged and undamaged) even though the natural frequencies of structures are used as both the independent and dependent variables. Moreover, the method developed in this paper has 225 better performances than the traditional method, while also eliminating the limitations of the methods proposed in the literature.

The rest of the paper starts with the methodology section where an introduction on regression analysis is given, followed by a detailed description of the damage detection method developed in this paper. The numerical beam model and the Z24 Bridge are then analysed using the ${ }^{230}$ damage detection methods. The paper then closes by drawing a set of conclusions.

\section{Methodology}

The damage detection method developed in this paper is described in this section. The method uses the regression analysis, therefore, a general overview of the regression analysis method in the context of damage detection is first given. Outlier analysis, which is commonly used to classify ${ }^{240}$ between undamaged and damaged for damage detection, is also briefly described in this section for completeness.

190 Finally, the damage detection method developed in this paper is described in details.

\subsection{Regression analysis}

Regression analysis is the study of the relationship between a set of independent and dependent variables 32 . The independent variables are characteristics that can be measured directly (e.g. temperature conditions) and these variables are used to predict the values of the dependent variables (e.g. natural frequency). Two general types of ${ }_{250}$ regression model exist; the simple regression model and the multiple regression model. In the simple regression model, there is only one independent variable and one dependent variable, while for the multiple regression model, several independent variables are used to obtain the dependent 255 variable.
In the context of damage detection, the independent variables used in the literature are usually the environmental and operational conditions (e.g. temperature and traffic loading), while the dependent variables are usually the vibration properties (e.g. natural frequencies) of structures. In this paper, it is proposed to use the natural frequencies of structures as both the independent and dependent variables, and to look for changes between the two sets of frequencies for damage detection. As mentioned previously, damage is a local phenomenon which affects each mode of vibration differently. By analysing the relationship between a pair of frequencies, damage alert can be raised when new observations do not follow the model of the undamaged structure. Therefore, the simple regression model is adopted in this paper to characterise the relationship between the frequencies of the undamaged structure, and is described below.

Consider the case where $y$ is the dependent variable and $x$ is the independent variable. In the regression analysis, a model is used to represent $y$ in terms of $x$. This means that, given a new value of $x$ and using the regression model, the value of $y$ can be predicted. The model of the simple linear regression is given below.

$$
y(x)=\alpha_{0}+\alpha_{1} x
$$

Where,

$\alpha_{0}$ is a coefficient, and

$\alpha_{1}$ is a coefficient defining the rate of change of $y$ based on $x$.

Eq. (1) is for the case where the relationship between $x$ and $y$ is linear (e.g. linear relationship between temperature and natural frequency). However, natural frequencies of civil engineering structures are subjected to both linear and nonlinear effects from the environmental and operational conditions [3, 6, 33. Therefore, the polynomial regression analysis has been adopted in the literature to better characterise the relationships between the environmental and operational conditions, and the natural frequencies of structures 33 . For example, Ding and Li 33 adopted several polynomial regression models to quantify the relationships between the natural frequencies of the Runyang Suspension Bridge and the temperature conditions. The polynomial regression is an extension of the linear regression and is given below 32 .

$$
y(x, n)=\alpha_{0}+\alpha_{1} x^{1}+\alpha_{2} x^{2}+\ldots+\alpha_{n} x^{n}
$$

where,

$y$ is the dependent variable,

$x$ is the independent variable,

$\alpha$ are coefficients of the model, and

$n$ is the $n^{\text {th }}$ degree of the polynomial.

The polynomial regression fits a nonlinear model to the independent and the dependent variables data set. As can be seen in Eq. 2), to fit the nonlinear model, the polynomial regression uses an $n^{\text {th }}$ degree of polynomial in $x$. It is an addition of terms (degree of polynomial) to the 
linear model. If a linear regression is adopted, only the first two terms on the right hand-side of Eq. (2) are kept. In this paper, the coefficients of the model are obtained ${ }_{310}$ using the least squares.

To prevent over-fitting the regression model, the polynomial order of the model is determined using the change in R-Squared $\left(R^{2}\right)$. The R-Squared is a statistic that explains the amount of variance accounted for in the rela-315 ionship between two (or more) variables 34. Therefore, the R-Squared gives an indication of the goodness of the model to represent the data.

In this paper, only when the change in R-Squared between two consecutive polynomial orders is less than $2 \%$ of the value of the R-Squared of the previous polynomial order that the increase in order should stop.

\subsection{Outlier analysis}

For damage detection, an outlier analysis is commonly ${ }^{325}$ used to classify between undamaged and damaged cases. set of control limits (usually an upper and a lower control limits) that define the range of variations of vibration properties (e.g. natural frequency) due to normal condi- ${ }^{330}$ tions (e.g. undamaged structure with normal environmenservation outside the control limits can then be labeled as an observation obtained from the damaged state.

To compute the control limits, the mean plus/minus ${ }^{335}$ three standard deviations approach, which is commonly used for damage detection [23, 31], is adopted in this paper. The control limits are given in Eq. (3).

$$
\begin{aligned}
U C L & =\mu+3 \sigma \\
L C L & =\mu-3 \sigma
\end{aligned}
$$

Where,

$U C L$ is the upper control limit calculated using a training database,

$L C L$ is the lower control limit calculated using a training database,

$\mu$ is the mean of the training database, and

$\sigma$ is the standard deviation of the training database.

It should be noted that the training database needs to be obtained from the normal (undamaged) condition of the structure.

\subsection{Graphical representation of damage detection meth- ods}

Graphical representations of the traditional regression ${ }_{355}$ analysis approach and the multivariate statistical tools approach for damage detection adopted in the literature are given in Fig. 1. In the traditional regression analysis approach, the environmental and operational parameters are required and are used as the independent variables. In $\operatorname{In}_{360}$

305 Fig. 1(a), the environmental and operational parameter adopted is the temperature conditions while the vibration property is the natural frequency. When damage occurs, the natural frequency will change, therefore, the deviation between the predicted value of natural frequency based on the temperature condition and the one obtained from field measurements for the same temperature condition, is used to indicate the presence of damage as shown in Fig. 11(a).

For the multivariate statistical tools approach (e.g. the use of Principal Component Analysis), the environmental and operational parameters are not required. The vibration properties are the only parameters required and in this example, the first two natural frequencies are used (Fig. 1(b)). It should be noted that this is only a graphical representation and usually more modes of vibration are analysed simultaneously, which creates more dimensions to the data set. In this approach, the multivariate statistical tools extract new features to represent the similarities and differences in the natural frequencies data set. For example, the similarity is the change in frequencies due to temperature effects as shown in Fig. 11(b), while the difference in the data set is the deviation of the damaged cases from the undamaged cases. Analysing the feature that represents the direction of the difference between the undamaged and damaged observations, damage detection can be achieved. It should be noted that the direction of the feature that represents the difference between the undamaged and damaged observations is perpendicular to the direction of the feature that represents the similarity in the data set.

In this paper, the regression analysis approach is combined with the principle behind the multivariate statistical tools approach for damage detection. The similarities and differences in the vibration properties data set can be extracted using the regression analysis in a more simple way as shown in Fig. 2. The change in natural frequency of the dependent variable due to damage for the same value of natural frequency of the independent variable, is analysed for damage detection. More information on the damage detection method developed in this paper is given in the next section.

\subsection{Damage detection method}

As mentioned previously, natural frequencies of structures are usually affected by the changing environmental and operational conditions, and usually a correlation (linear or nonlinear) exists between the frequencies and the different environmental and operational parameters. Since the values of the natural frequencies depend on the environmental and operational parameters, in the traditional regression damage detection methods, the frequencies are used as the dependent variables while the environmental and operational conditions are used as the independent variables. Consider the case where temperature is the environmental effect affecting the natural frequencies of a structure. The relationships between temperature (independent variable) and the first two natural frequencies (dependent variables) are assumed to be linear and are given in Eq. (4). It should be noted that Eq. (4) is for the case where the structure is not subjected to damage, therefore, 
(a)

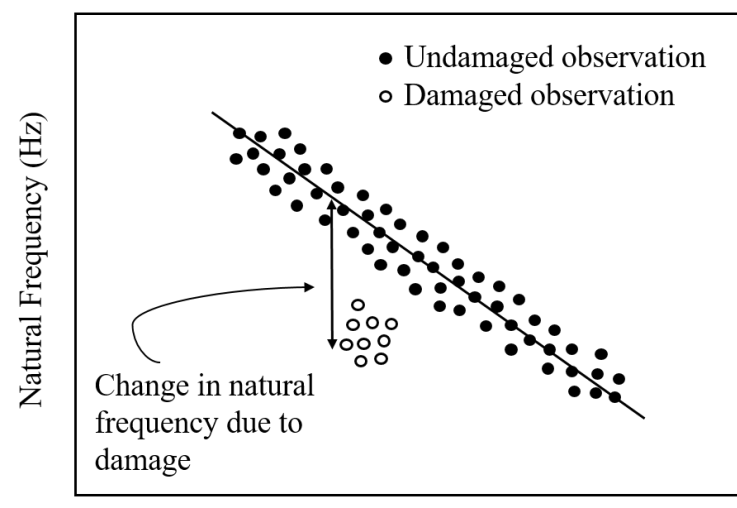

Temperature

Traditional regression analysis approach (b)

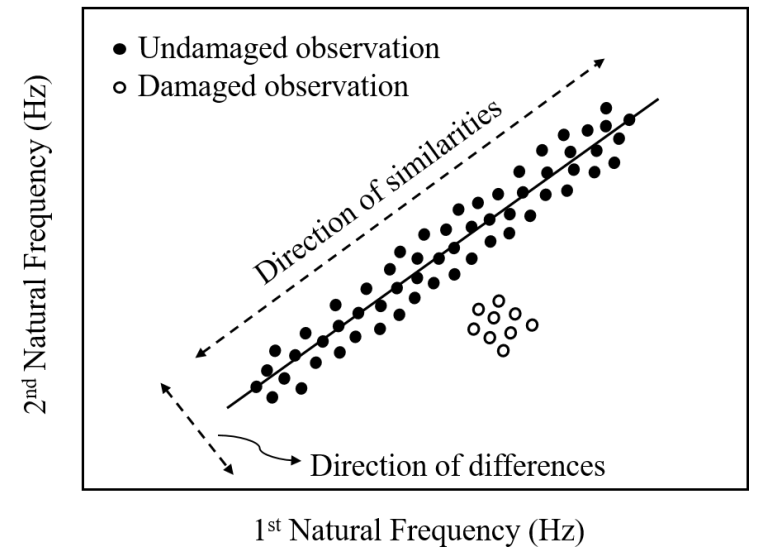

Traditional multivariate statistical approach

Figure 1: Graphical representation of (a) traditional regression analysis approach and (b) traditional multivariate statistical tools approach for damage detection.

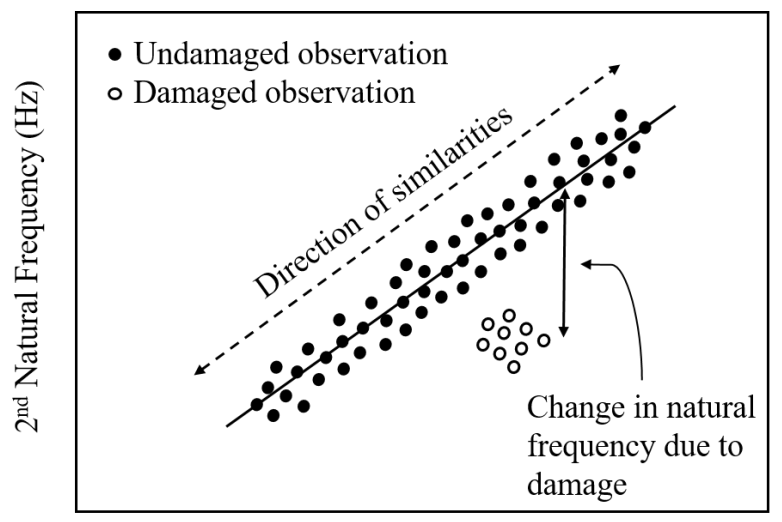

$1^{\text {st }}$ Natural Frequency $(\mathrm{Hz})$

Figure 2: Graphical representation of the damage detection method proposed in this paper.

the natural frequencies are the frequencies of the healthy state of the structure.

$$
\begin{aligned}
& f_{1, p}=f_{1}+\alpha T_{p} \\
& f_{2, p}=f_{2}+\beta T_{p}
\end{aligned}
$$

Where,

$f_{1}$ represents the base value of the first natural frequency (e.g. value of natural frequency at $0{ }^{\circ} \mathrm{C}$ ),

$f_{2}$ represents the base value of the second natural fre- ${ }^{400}$ quency (e.g. value of natural frequency at $0{ }^{\circ} \mathrm{C}$ ),

$f_{1, p}$ represents the value of the first natural frequency of the $p^{t h}$ observation,

$f_{2, p}$ represents the value of the second natural frequency of the $p^{t h}$ observation,

$T_{p}$ represents the temperature condition of the $p^{t h}$ observation, $\alpha$ is a coefficient which defines the relationship between the first natural frequency and temperature, and

$\beta$ is a coefficient which defines the relationship between the second natural frequency and temperature.

From Eq. (4), it can be seen that both frequencies are dependent upon the same independent variable, the temperature condition. These two frequencies can therefore be related to each other as follows.

$$
f_{2, p}=f_{2}+\beta \frac{\left(f_{1, p}-f_{1}\right)}{\alpha}
$$

Eq. (5) is the model representing the relationship between the first and second natural frequencies. In Eq. (5), the first natural frequency is the independent variable while the second natural frequency is the dependent variable. Therefore, the value of the second natural frequency can be predicted based on the value of the first natural frequency. As mentioned previously, when damage occurs in a structure, each mode of vibration will be affected differently, which will create a different model (different model to the model of the undamaged structure) of the frequencies. Thus, this difference can be used for damage detection, similar to the multivariate statistical tools approach (Fig. 1(b)) proposed in the literature.

In this paper, it is proposed to use the natural frequencies as both the independent and dependent variables. It is then proposed to use the difference between the predicted value of natural frequency of the dependent variable and the value of the dependent variable obtained from field measurements, as a deviation index for damage detection (Fig. 2).

$$
\text { Deviation Index }=f_{2, p}-f_{2, p, f}
$$

Where, $f_{2, p, f}$ is the value of the second natural frequency of the $p^{\text {th }}$ observation obtained from field mea- 
surements. If the structure is in healthy condition and subjected to temperature effect only, the deviation index 465 will be zero, while if the structure is subjected to damage, the deviation index will not be zero. For real-life structures, the deviation index of the undamaged state of the structures is usually not zero because other environmental and operational conditions (e.g. traffic loading), noise and ${ }_{470}$ processing errors will deviate the values of the frequencies from the ideal values (ideal values due to temperature effect only). These cases will have a deviation index with small magnitude while the damaged cases will have larger magnitudes.

To classify whether the cases are from the healthy or damaged state of the structure, it is proposed to perform an outlier analysis on the deviation index. Any future observation outside the control limits can then be attributed to damage.

Usually, several modes of vibration are extracted, therefore, several pairs of natural frequencies can be used to create the regression model for damage detection. The results of each pair of natural frequencies can be combined together and the damage alert is raised when the observation is outside the control limits in at least one of the pairs of frequencies. In this paper, the first four natural frequencies will be analysed since usually a minimum of the first four natural frequencies are extracted from reallife structures and analysed [3, 8, 11].

A multiple regression model could have been adopted for damage detection instead of several simple regression models. However, the problem with using only one multiple regression model is that, it is difficult to determine the dependent variable. As mentioned previously, each mode of vibration is affected to a different degree by damage. To maximise the performance of the method developed and to avoid false alerts, it is ideal to create a model between the least affected and the most affected modes of vibration, thus, one of these modes need to be the dependent variable. However, this is difficult to determine because dif-

445 ferent damage scenarios (different damage locations and extent) affect the modes of vibration differently. Therefore, creating a model for each pair of natural frequencies will maximise the performance of the method developed in this paper while also reducing false alerts.

To summarise, it is proposed to first capture natural frequencies from the undamaged state of the structure that needs to be monitored, followed by creating regression ${ }_{480}$ models (linear or nonlinear models) between pairs of natural frequencies. To choose the polynomial model order, the

455 change in R-Squared between two consecutive polynomial orders is used. In the regression model, one mode of vibration is used as the independent variable while the other ${ }_{485}$ mode of vibration is used as the dependent variable. New measurements can then be compared to the model created. The value of the natural frequency of the dependent variable can be predicted based on the value of the independent variable obtained from field measurements. The difference between the predicted value of the dependent ${ }_{490}$ variable and the value of the dependent variable obtained from field measurements can then be used as a deviation index and analysed using an outlier analysis. If the deviation index is larger than the normal situation, then the damage alert is raised. Since several regression models are created for different pairs of frequencies, the damage alert is raised when the new monitored observation is outside the control limits in at least one of the model. To compute the control limits for the oulier analysis, the training database adopted is from the measurements used to create the regression models. A flow chart summarising the procedures to follow for damage detection is also given in Fig. 3 .

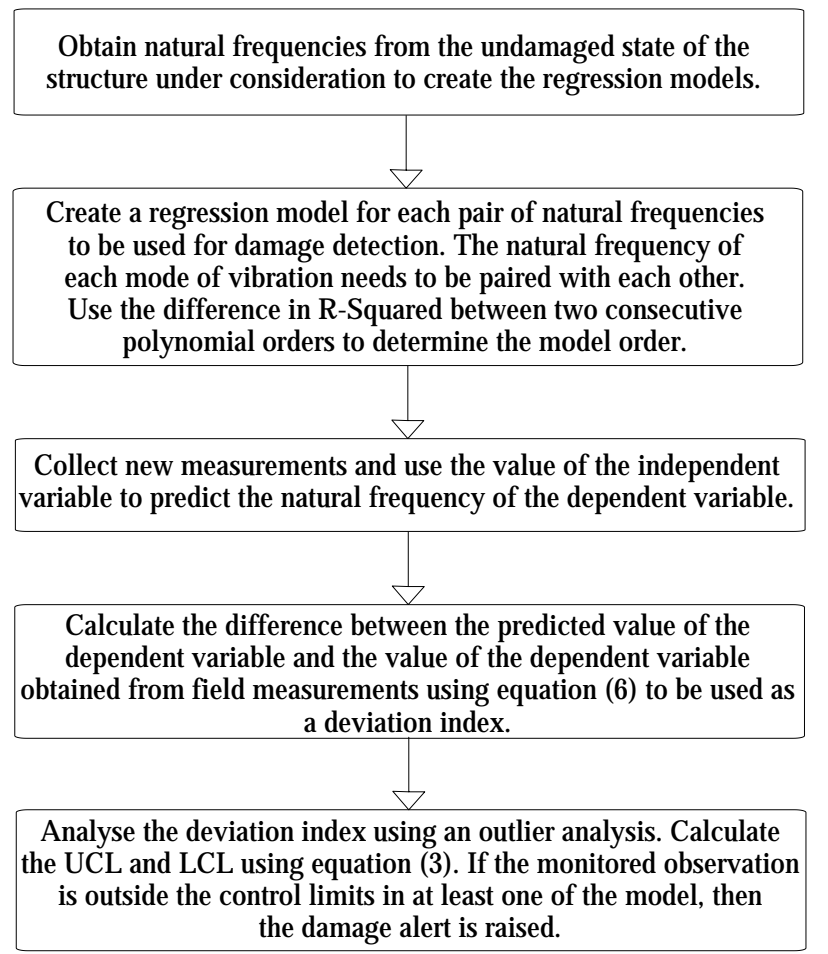

Figure 3: Procedures to follow for damage detection.

\section{Case studies}

To illustrate the application of the method developed in this paper, two case studies are examined in this section. The first is a numerical beam model which is subjected to changing temperature conditions and to varying mass distribution. The second is a real-life bridge structure, the Z24 Bridge, in Switzerland, which was subjected to complicated environmental and operational conditions. The traditional regression analysis method is also applied to the numerical beam model and the Z24 Bridge for comparison with the method developed in this paper.

\subsection{Beam structure model}

The beam structure model under consideration is presented in Fig. 4 and is subjected to changing temperature 
conditions and to varying mass distribution. The structure ${ }_{520}$ is $10 \mathrm{~m}$ long and consists of ten beam elements of $1 \mathrm{~m}$ each. The cross-sectional area and second moment of area of the structure are $0.08 \mathrm{~m}^{2}$ and $0.0006 \mathrm{~m}^{4}$, respectively. To simulate a varying environmental condition, the Young's modulus of the material is assumed to be temperature de-525 pendent. The relationship between the Young's modulus of the material and temperature is given in Fig. 5 and is the same as the one assumed by Kullaa 35. It should be noted that in reality, such a Young's modulus-temperature relationship may not exist. It is adopted here to simulate ${ }_{530}$ the changes in natural frequencies due to the effects of temperature and the nonlinear relationships between the frequencies and temperature that are commonly found for real-life structures such as the Z24 Bridge 3 and the Meriden Bridge [6]. For real-life structures, a combination of $f_{535}$ effects such as the change in boundary conditions due to thermal effects and the different materials that the structures are composed of will define the vibration properties due to temperature effects. The density of the material is assumed to be $7850 \mathrm{~kg} / \mathrm{m}^{3}$.

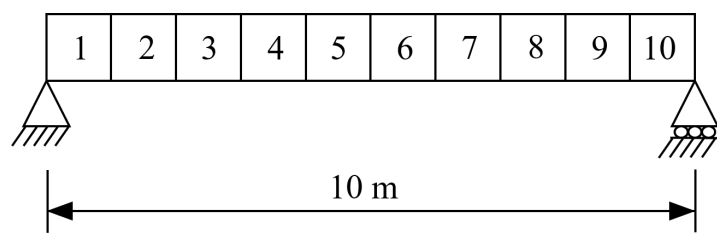

Figure 4: Beam structure model.

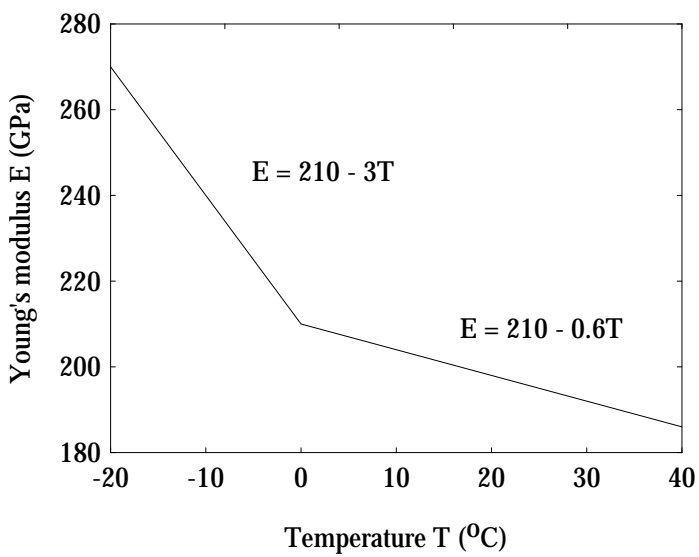

Figure 5: Variations of Young's modulus with temperature for the beam structure model.

The first four natural frequencies of the beam are analysed using the damage detection method developed in this paper. The natural frequencies of 3000 observations from the undamaged state of the structure are adopted to construct the regression model used for damage detection. The temperature conditions of these 3000 observations are uniformly distributed between $-20{ }^{\circ} \mathrm{C}$ and $40{ }^{\circ} \mathrm{C}$. Another 1000 observations obtained from the undamaged state of the structure are used to validate the models created. For testing, another 600 observations from the undamaged state of the structure are analysed along with 900 damaged cases. For the damaged cases, the stiffness of the $4^{\text {th }}$ element is reduced. Three different damage levels are considered: $20 \%, 25 \%$ and $30 \%$ reduction in stiffness. The range of temperature conditions of these cases (undamaged and damaged cases analysed for damage detection) is also between $-20{ }^{\circ} \mathrm{C}$ and $40{ }^{\circ} \mathrm{C}$. It should be noted that the distribution of the temperature conditions (uniformly distributed) adopted in this case study is not that important because for this regression analysis damage detection method, the deviation away from the regression model is important, not the deviation in the direction parallel to the model (direction of distribution of temperature conditions is parallel to the model).

In addition to the effect of temperature, the structure is also subjected to variations in mass to simulate pedestrian loading or traffic loading that can be present in a building or on a bridge structure similar to Soo Lon Wah et al. 31 . To simulate the variations in mass, the beam elements are subjected to a $\pm 5 \%$ and a $\pm 7.5 \%$ range of uniform variations in density. Two different range of variations are used to create some outlier measurements (e.g. the \pm 7.5 $\%$ range creates larger variations which can be due to some heavy weight vehicles or due to monitoring and processing errors while extracting the natural frequencies) in the data set to create more realistic scenarios. Therefore, the 3000 observations data set is separated into two data sets. The first set consists of 2000 observations with a $\pm 5 \%$ range of variations in density while the second set consists of 1000 observations with a $\pm 7.5 \%$ range of variations in density. The cases analysed are also subjected to the two different range of variations in density.

The plot of the first four natural frequencies of the beam structure against temperature conditions is shown in Fig. 6 for the 3000 undamaged observations (black dots) and the damaged observations (red dots) used for testing. For the damaged observations, only observations with temperature conditions between $10{ }^{\circ} \mathrm{C}$ and $20{ }^{\circ} \mathrm{C}$, and $-5{ }^{\circ} \mathrm{C}$ and $-15{ }^{\circ} \mathrm{C}$ are included in the figure. This is because, if all the damaged observations were included, the undamaged observations would be hidden in some of the plots similar to Fig. 6(c). It can be seen in Fig. 6 that a bilinear relationship exists between the temperature conditions and the natural frequencies of the undamaged cases of the structure. Moreover, it can be seen that the third mode of vibration (Fig. 6(c)) is relatively unaffected by the presence of damage, while the other modes of vibration are more affected. Therefore, this difference can be used for damage detection. The third mode of vibration is relatively unaffected because the damage location is near a node of the third mode.

The damage detection method developed in this paper is applied to the data of this beam structure model. Regression models of pairs of natural frequencies are first created. The first, second, third and fourth natural fre- 
(a)

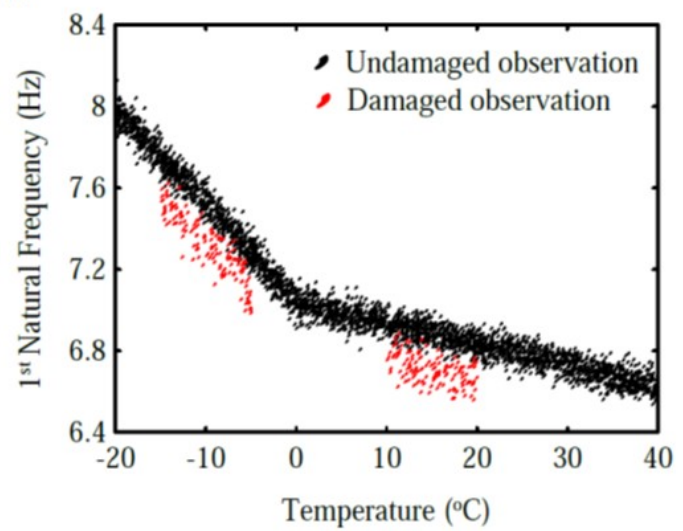

(c)

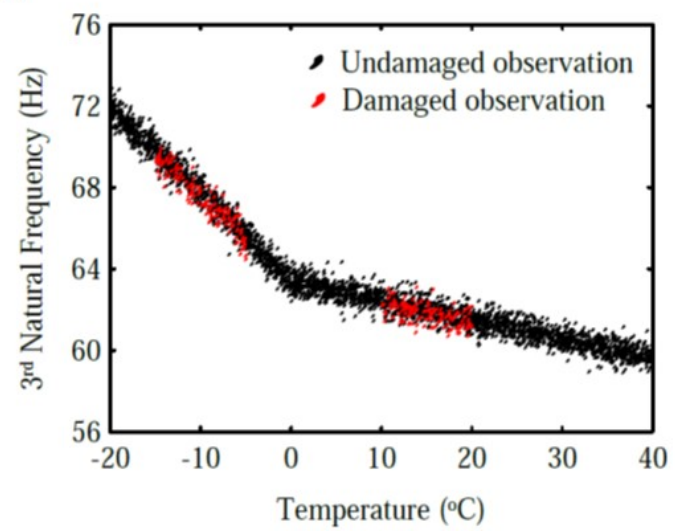

(b)

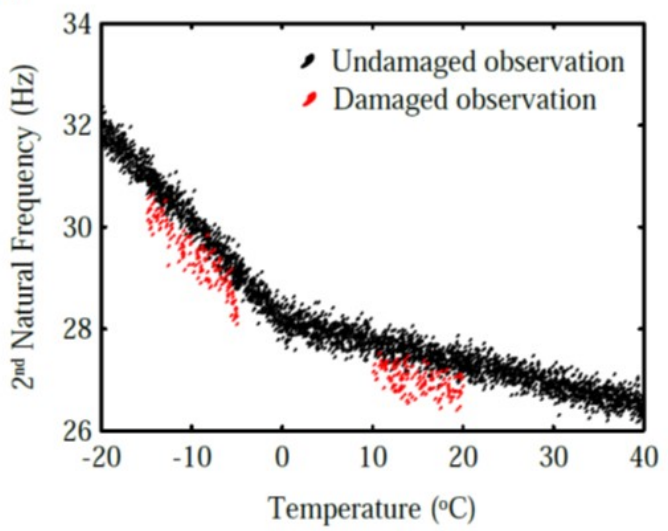

(d)

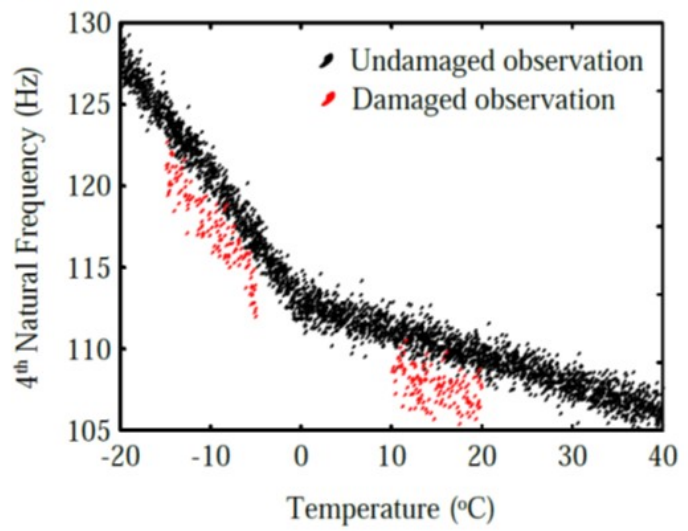

Figure 6: Plot of temperature versus (a) $1^{\text {st }}$ natural frequency, (b) $2^{\text {nd }}$ natural frequency, (c) $3^{\text {rd }}$ natural frequency and (d) $4^{\text {th }}$ natural frequency of the 3000 undamaged observations and the damaged observations (only for temperatures of $10^{\circ} \mathrm{C}$ to $20^{\circ} \mathrm{C}$ and $-5{ }^{\circ} \mathrm{C}$ to $-15^{\circ} \mathrm{C}$ ) of the beam structure model.

quencies are used as the independent and dependent vari-600 ables. Each natural frequency needs to be paired with each other, therefore, six pairs of frequencies are created $1 \mathrm{NF}$ vs $2 \mathrm{NF}, 1 \mathrm{NF}$ vs $3 \mathrm{NF}, 1 \mathrm{NF}$ vs $4 \mathrm{NF}, 2 \mathrm{NF}$ vs $3 \mathrm{NF}, 2 \mathrm{NF}$ vs $4 \mathrm{NF}$ and $3 \mathrm{NF}$ vs $4 \mathrm{NF}$, where NF represents natural frequency). Each natural frequency is paired with 605 each other so as maximise the performance of the method by having a model containing the least affected and the most affected modes of vibration for any damage state. The plot of each pair of natural frequencies is given in Fig. 7 for the 3000 undamaged observations (black dots) and 610 the damaged observations (red dots for temperature conditions between $10{ }^{\circ} \mathrm{C}$ and $20{ }^{\circ} \mathrm{C}$, and $-5{ }^{\circ} \mathrm{C}$ and $-15{ }^{\circ} \mathrm{C}$ only) used for testing. From the plots, it can be seen that when the third natural frequency is present in the pair of frequencies (Figs. $7(\mathrm{~b}, \mathrm{~d}$ and f) ), the damaged observa-615 tions cluster separately from the undamaged observations. However, when the third natural frequency is not present (Figs. 7(a, c and e)), the undamaged and damaged observations cluster together. Although the natural frequencies are used as both the independent and dependent variables, 620 a clear distinction can be seen between the undamaged and damaged observations. This is because, as mentioned pre- viously, each mode of vibration is affected differently by damage, hence, this creates a change in relationship between the frequencies, from the undamaged conditions.

After the natural frequencies have been paired with each other, the regression models need to be created. To determine the polynomial order for each model, the difference in R-Squared between two consecutive polynomial orders, is analysed. Only when the difference in R-Squared between two consecutive polynomial orders is less than 2 $\%$ of the R-Squared value of the lower polynomial order that the increase in order will stop. For this beam structure, the linear regression model is adopted for all pairs of frequencies because the percentage change in R-Squared is almost $0 \%$ between any two consecutive polynomial orders.

After the polynomial orders have been chosen and the models created, each model is used to predicted the natural frequencies of the dependent variable of new observations based on the natural frequencies of the independent variable obtained from the structure. The difference between the predicted value of the dependent variable and the value of the dependent variable obtained from the structure is used as a deviation index. An outlier analysis is then ap- 
(a)

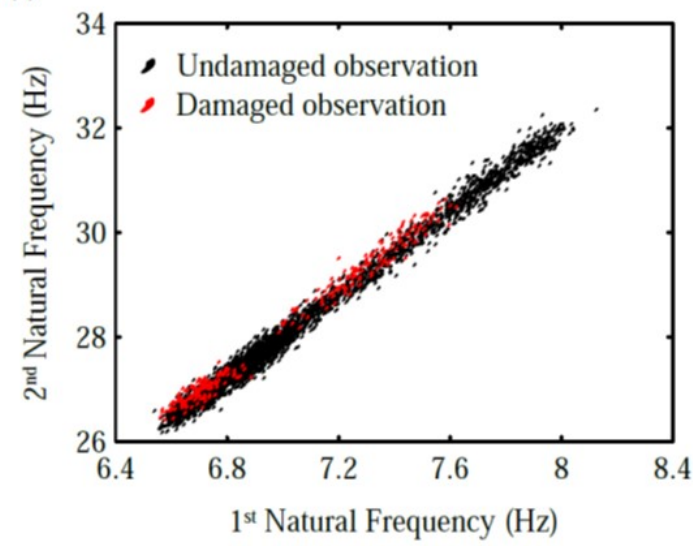

(c)

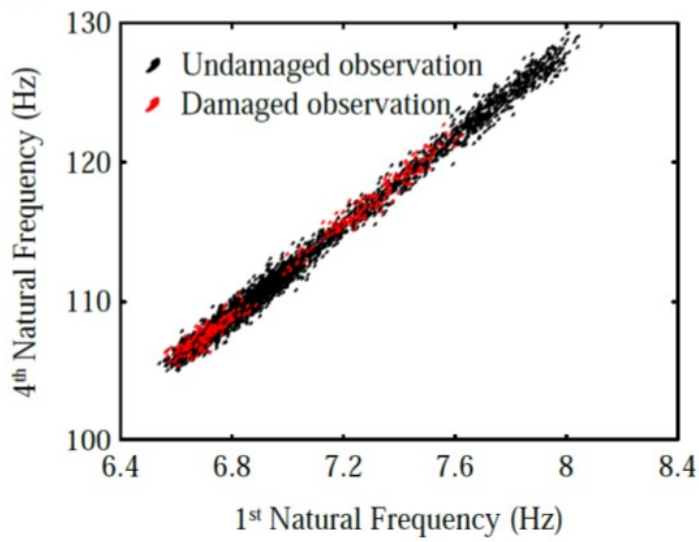

(e)

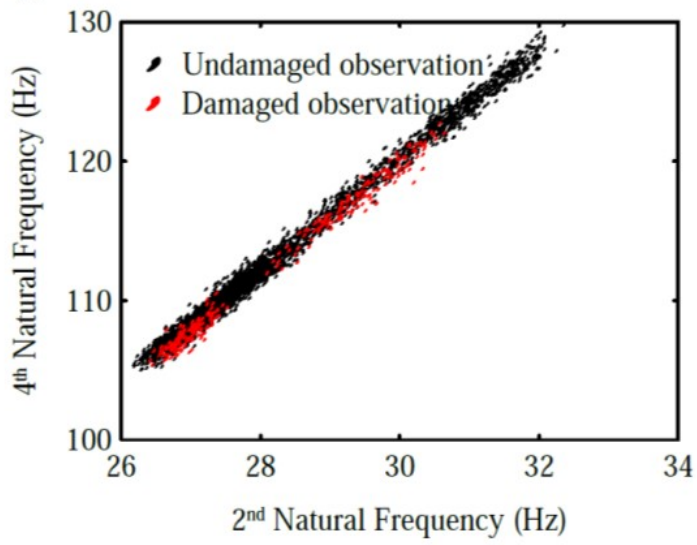

(b)

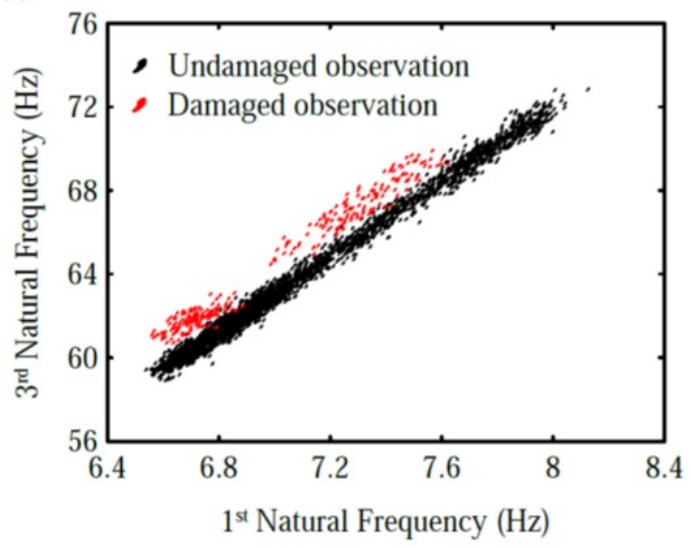

(d)

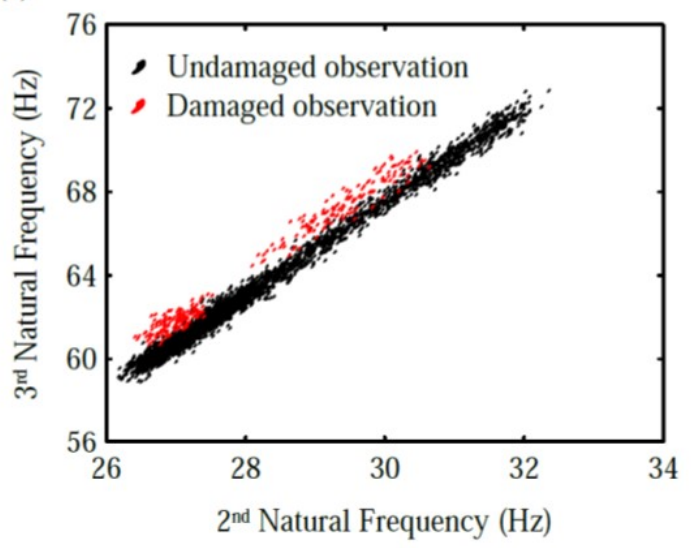

(f)

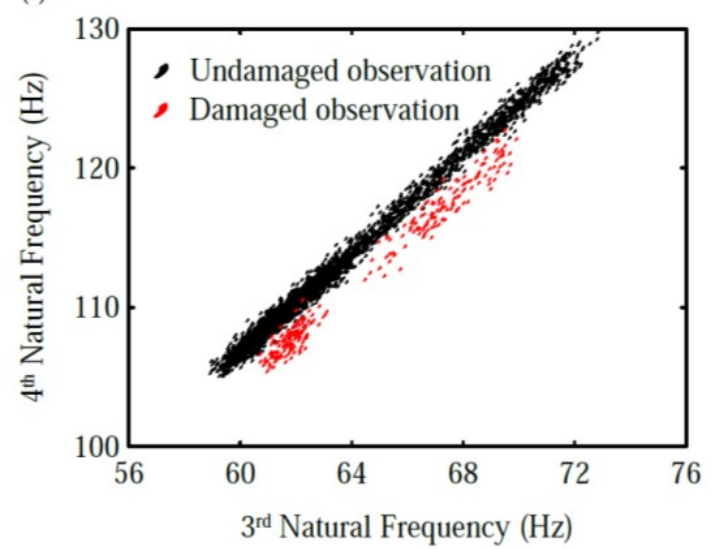

Figure 7: Plot of (a) $1^{\text {st }}$ vs $2^{\text {nd }}$ natural frequency, (b) $1^{\text {st }}$ vs $3^{\text {rd }}$ natural frequency (c) $1^{\text {st }}$ vs $4^{\text {th }}$ natural frequency, (d) $2^{\text {nd }}$ vs $3^{\text {rd }}$ natural frequency, (e) $2^{\text {nd }}$ vs $4^{\text {th }}$ natural frequency and (f) $3^{\text {rd }}$ vs $4^{\text {th }}$ natural frequency of the 3000 undamaged observations and the damaged observations (only for temperatures of $10{ }^{\circ} \mathrm{C}$ to $20{ }^{\circ} \mathrm{C}$ and $-5{ }^{\circ} \mathrm{C}$ to $-15^{\circ} \mathrm{C}$ ) of the beam structure model.

plied on the deviation index to classify between undamaged and damaged states. To calculate the control limits for the outlier analysis, the deviation index obtained from the 3000 observations used to construct the regression models are used as the training database. Two con-635 trol limits (upper and lower control limits) are created for each pair of natural frequencies and are calculated using Eq. (3). Before the 600 undamaged and 900 damaged cases are analysed, the models and control limits created are first validated. 1000 observations (used as a database for validation) obtained from the undamaged state of the structure are analysed and the deviation index of these observations are compared to the control limits created. The deviation index of these observations lie within the control limits, indicating that the models created were able to represent the undamaged state of the structure. 
The results of the outlier analysis for the 600 undamand 900 damaged cases analysed are presented in Fig. 8. In Fig. 8, the dots represent the observations being analysed while the two dotted horizontal lines are the control limits (UCL and LCL). Three dotted vertical lines7oo are also included in the figure to separate the undamaged cevels of damage represented by D1, D2 and D3). From the plots it can be seen that most of the undamaged cases lie within the control limits indicating that the structure is in healthy condition. The 705 undamaged cases analysed lie within the control limits be, the fluctuation of the frequencies from the ideal values (ideal values due to temperature effects only) are only due to the variations in mass of the beam elements, which have a similar range of variations as the 3000 un-710 damaged observations used to create the control limits. No other external effects affect the structure and the natural frequencies.

For the damaged cases analysed, most of the observations lie outside the control limits when the third natural ${ }_{715}$ frequency is present in the model of the pair of frequencies

${ }_{660}$ (Figs. 8(b, d and f)). This indicates that the structure is damaged. These observations lie outside the control limits because the third natural frequency is less affected by the presence of damage than the first, second, and fourth fre-720 quencies. This difference creates a change in relationship

665 between the pair of frequencies from the regression model of the undamaged state of the structure, hence, clustering these damaged cases away from the undamaged observations (3000 undamaged observations). For the other mod-725 els of pair of natural frequencies (that does not include the third natural frequency), most of the damaged cases lie within the control limits (Figs. 8(a, c and e)). These results highlight the importance to analyse the relationship between the least affected and the most affected modes of $f_{730}$ vibration to avoid false alerts. It is important to create a model for each pair of frequencies.

To classify the cases, the results of the outlier analysis using the six models are grouped together and the damage alert is raised only when the monitored observation ${ }_{735}$ is outside the control limits in at least one of the model. The successful rates of alerting healthy condition for the undamaged cases and alerting damage for the damaged cases using the method developed in this paper are $97.7 \%$ and $94.4 \%$, respectively.

The mean of the deviation index of the four scenarios (undamaged and three levels of damage) analysed is also given in Fig. 8 using a bold horizontal line. In each scenario, the deviation index does not lie exactly on the mean value due to the effects of the variations of mass. The vari-745 ations of mass fluctuates the natural frequencies from their ideal values due to temperature effect only. For the undamaged cases, it can be seen that the mean is around the zero value. This is because, if the variations of mass was not present, the natural frequencies of these cases would 750 have fallen along the regression model (regression model of ios, it can be seen that when the third natural frequency is present in the pair of frequencies (Figs. 8(b, d and f)), almost all the mean values are outside the control limits. Only in Fig. 8(d) that the mean value of the first damage level is inside the limits. For these damaged scenarios, the fluctuation of the deviation index from the mean is also due to the variations of mass of the beam elements. It can also be seen from the figure that, the magnitude of the mean value becomes larger with the increase in damage extent. Therefore, in addition of detecting damage, the proposed method can also indicate damage progression.

The damage detection method proposed in this paper is applied again on the data of the beam structure model, but this time, the natural frequency of each mode of vibration is used as the independent variable and is paired with each of the other modes of vibration (used as dependent variable). Therefore, twelve regression models are constructed for damage detection. In the previous case study, only six regression models were used because six pairs of natural frequencies are enough to match all four modes of vibration with each other. Similar to the previous case study, the 3000 undamaged observations are used to create the regression models, and the 600 undamaged and 900 damaged cases are analysed. The successful rates of alerting healthy condition for the undamaged cases and alerting damage for the damaged cases are $97.7 \%$ and 95 $\%$, respectively. The successful rate for the undamaged cases is the same as the previous case study while for the damaged cases, an improvement of $0.6 \%$ is obtained when the twelve models are used. This can be attributed to the fact that all four modes of vibration are used as the dependent variable, while in the previous case study, the first natural frequency was not used as the dependent variable. Using all four modes of vibration allow the change in natural frequencies due to damage to be obtained for all modes of vibration.

Since the method developed in this paper uses the regression analysis, the data of this beam structure is also analysed using the traditional regression damage detection method for comparison. The temperature condition is used as the independent variable while the four natural frequencies are used as the dependent variables. Four regression models are created for damage detection, one for each natural frequency. Similar to the method developed in this paper, the polynomial order of each model is determined by analysing the difference in R-Squared between two consecutive polynomial order models. The percentage difference in R-Squared for the first six polynomial orders are given in Table 1 for all four natural frequency-temperature conditions models. The polynomial order adopted for each model is also highlighted in Table 1 using the symbol '*'. For example, a cubic polynomial order is adopted for all four models because the percentage change in R-Squared between the third and fourth degrees of polynomial is almost zero. In this paper, only when the difference in RSquared between two consecutive polynomial orders is less than $2 \%$ of the R-Squared value of the lower polynomial 
(a)

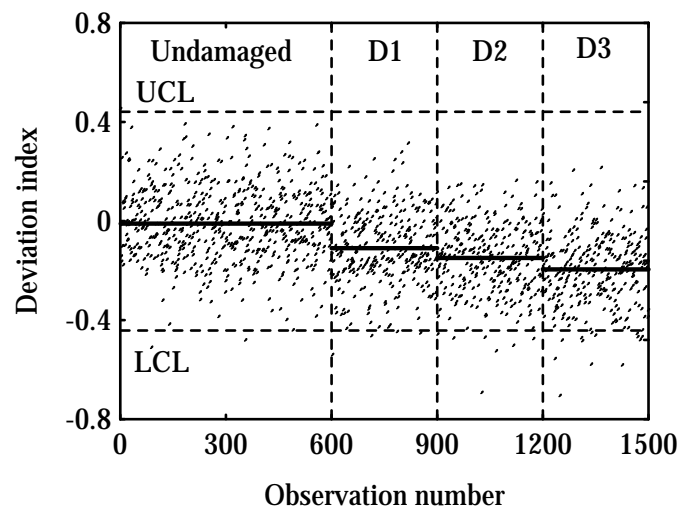

Independent variable: $1 \mathrm{NF}$, D ependent variable: $2 \mathrm{NF}$

(c)

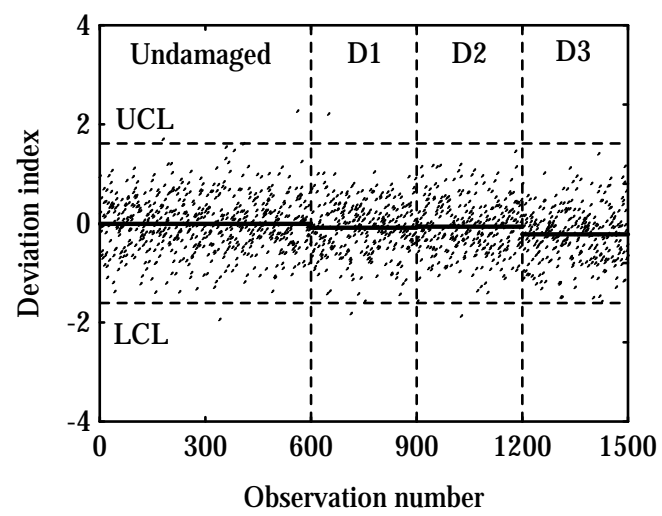

Independent variable: $1 \mathrm{NF}$, Dependent variable: 4NF

(e)

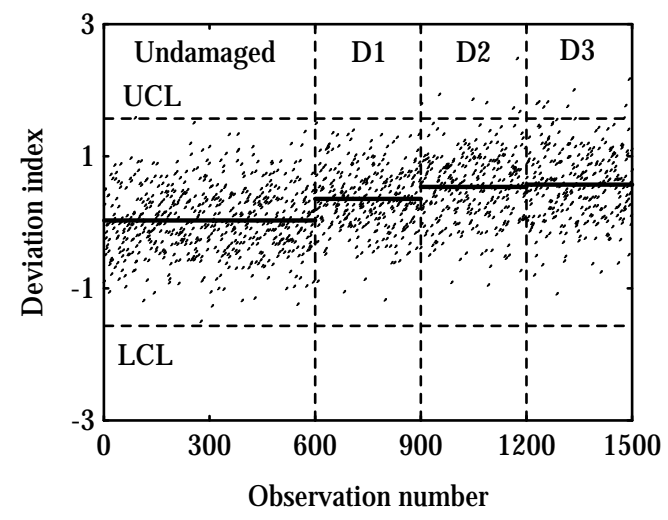

Independent variable: 2NF, Dependent variable: 4NF (b)

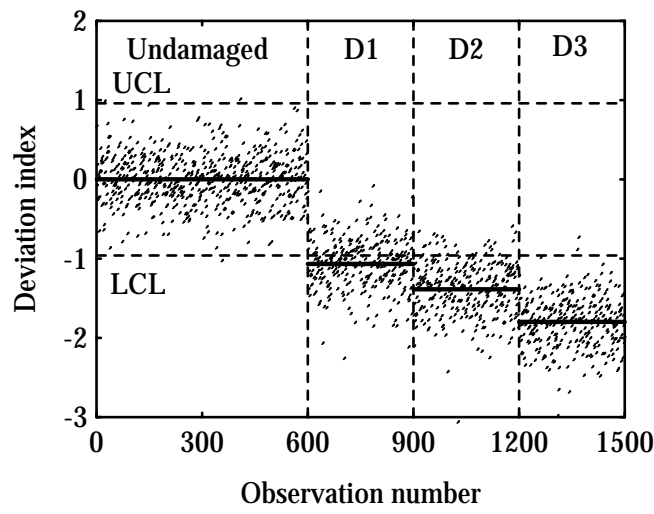

Independent variable: $1 \mathrm{NF}$, Dependent variable: 3NF

(d)

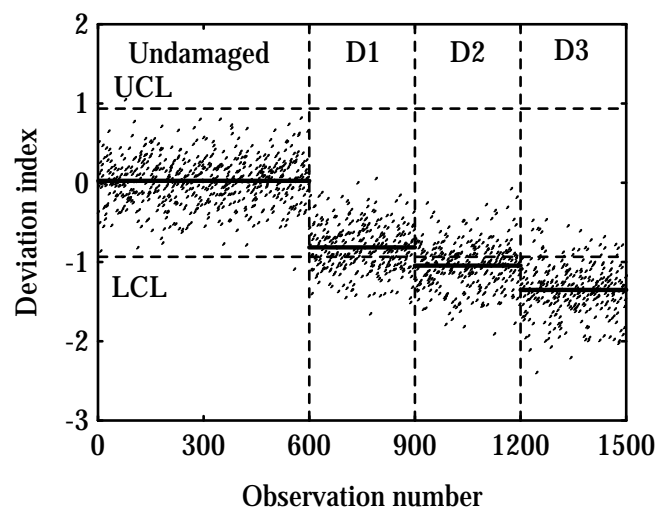

Independent variable: 2NF, Dependent variable: 3NF

(f)

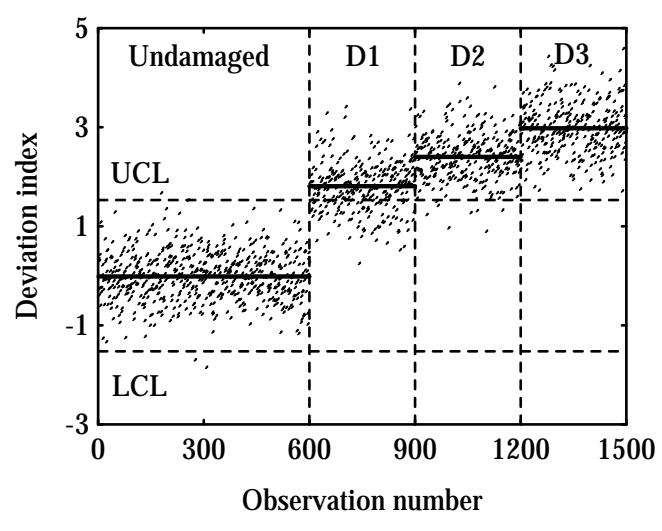

Independent variable: 3NF, Dependent variable: 4NF

Figure 8: Results of outlier analysis of the beam structure model using the method developed in this paper. ${ }^{*}$ NF represents natural frequency

order that the increase in order will stop. The models are 60 also validated by analysing the 1000 undamaged observations.

After the regression models are created, the natural frequencies of new observations are predicted based on their temperature conditions. The difference between the pre-765 dicted value of natural frequencies and the one obtained from the structure for the same temperature condition is used as a deviation index. An outlier analysis is then adopted to classify the cases and the results obtained are presented in Fig. 9. From Fig. 9, it can be seen that most of the undamaged cases lie within the control limits, indicating that no damage is present in the beam structure. From Fig. 9(c), it can also be seen that most of the 
Table 1: Percentage change in R-Squared from previous polynomial order. Chosen polynomial order highlighted with the symbol '*'.

\begin{tabular}{|c|c|c|c|c|}
\hline Polynomial order & $1^{\text {st }}$ Natural Frequency & $2^{\text {nd }}$ Natural Frequency & $3^{\text {rd }}$ Natural Frequency & $4^{t h}$ Natural Frequency \\
\hline 1 & - & - & - & - \\
\hline 2 & 13 & 13 & 12 & 13 \\
\hline 3 & $2^{*}$ & $2^{*}$ & $2^{*}$ & $2^{*}$ \\
\hline 4 & 0 & 0 & 0 & 0 \\
\hline 5 & 0 & 0 & 0 & 0 \\
\hline 6 & 0 & 0 & 0 & 0 \\
\hline
\end{tabular}

(a)

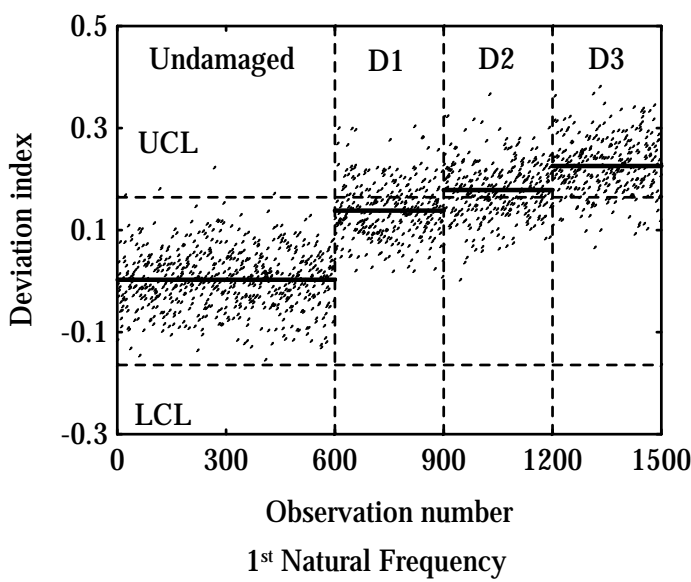

(c)

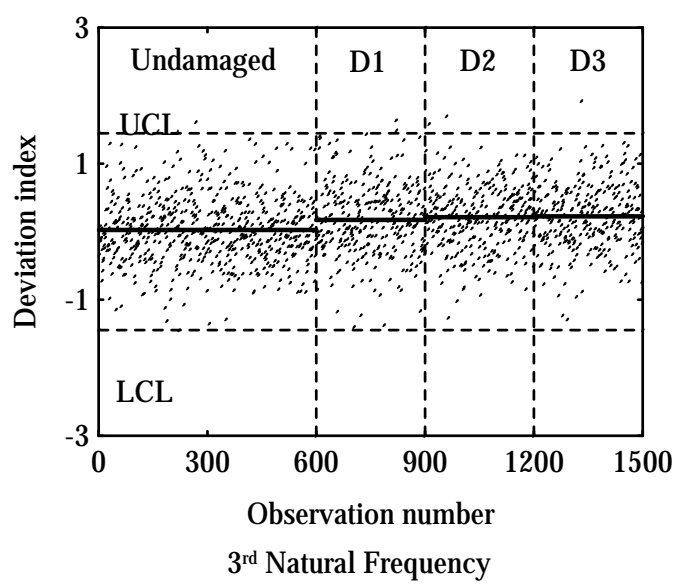

(b)

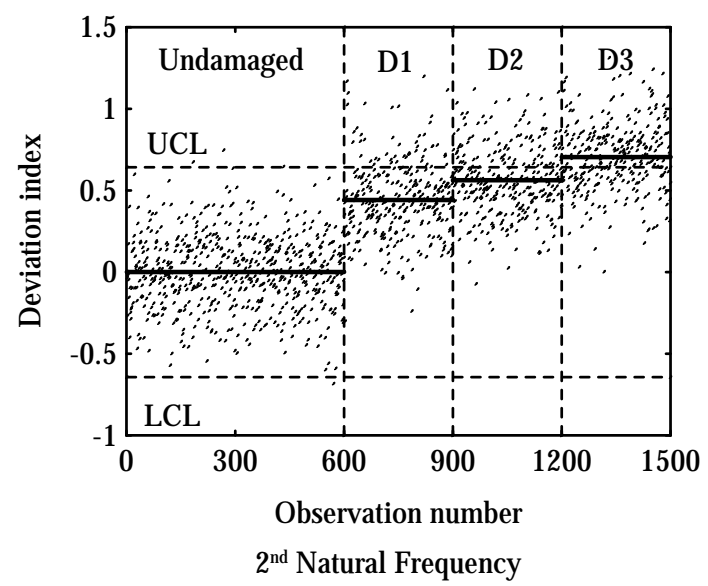

(d)

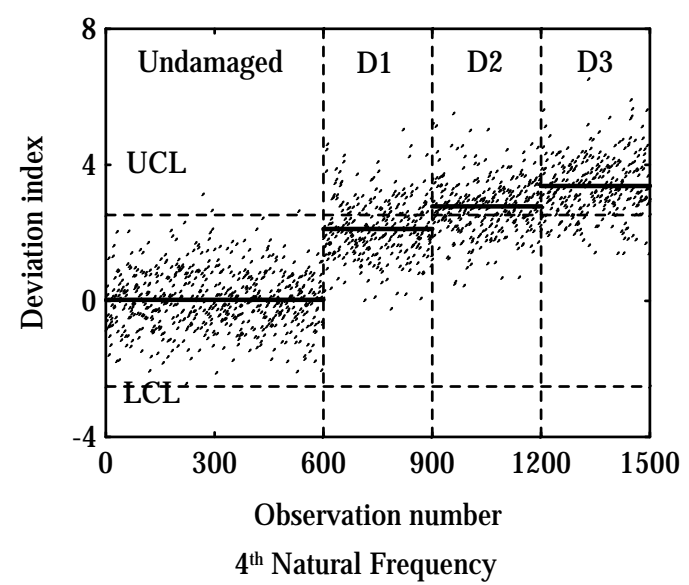

Figure 9: Results of outlier analysis of the beam structure model using the traditional regression damage detection method with the dependent variable being the (a) $1^{\text {st }}$ natural frequency, (b) $2^{\text {nd }}$ natural frequency, (c) $3^{\text {rd }}$ natural frequency and (d) $4^{\text {th }}$ natural frequency.

damaged cases lie within the control limits indicating that no damage is present in the structure. These damaged observations lie within the control limits because as men-780 tioned previously, the third mode of vibration of this structure is relatively unaffected by the presence of damage. Therefore, the fluctuation in the third natural frequency is mainly attributed to the temperature effect and to the variations in mass effect which is similar to the undamaged ${ }_{785}$ cases. For the first, second and fourth natural frequencies, some of the damaged observations are outside the control limits, indicating that damage is present. Similar to the method developed in this paper, the damage alert is raised only when the monitored observation is outside the control limits in at least one of the model. The successful rates for the undamaged and damaged cases analysed are 98.3 $\%$ and $67.9 \%$, respectively.

From the results obtained using the two damage detection methods, it can be concluded that the method developed in this paper performs better than the traditional regression damage detection method for the damaged cases with an improvement of almost $27 \%$. Even though the natural frequencies are used as both the independent and 
dependent variables, the damaged cases are well classified. tained using the traditional regression damage detection method. The undamaged observations that are alerted as damage may be the observations that are subjected to higher variations in mass $( \pm 7.5 \%)$ to simulate the effects undamaged observations with high noise level and measurements error as damage, a method will be introduced in the next section where a real-life bridge structure, the Z24 Bridge, will be analysed.

800 The damage detection method developed in this paper and the traditional regression damage detection method are applied again to the data of the beam structure, but this time, measurements from limited environmental conditions are used to construct the regression models. Only measurements with temperature conditions between -10 ${ }^{\circ} \mathrm{C}$ and $20{ }^{\circ} \mathrm{C}$ are used for the regression models. These measurements are obtained from the database of the 3000 undamaged measurements. All the 600 undamaged and 900 damaged cases which were obtained under a range of 810 temperature conditions of $-20^{\circ} \mathrm{C}$ to $40^{\circ} \mathrm{C}$ are used for testing. The successful rates of alerting healthy condition for the undamaged cases and damage for the damaged cases using the method developed in this paper are $96.7 \%$ and $96.6 \%$, respectively. It should be noted that only six mod-

815 els are used for damage detection (same pairs of natural frequencies as the first case study). For the traditional regression damage detection method, $59.7 \%$ of the undamaged cases are correctly classified, while $82.3 \%$ of the damaged cases are identified as being damaged. From the results obtained using the method developed in this paper, it can be seen that almost the same successful rates are obtained when the full range and the limited range of environmental conditions are used to create the regression models. For the traditional regression damage detection method, better results are obtained when the full range of environmental conditions is used. It should be noted that, although similar results are obtained when a smaller range of environmental conditions is used with the damage detection method developed in this paper, it is ideal to use measurements obtained from a wide range of environmental and operational conditions to construct the regression models. This will allow the models to represent all pos-855 sible conditions the structure may encounter, which can reduce false alerts.

\subsection{Z24 Bridge}

The Z24 Bridge (Fig. 10, a post-tensioned concrete cho $^{860}$ box girder bridge, was located in Switzerland connecting Koppigen and Utzenstorf and overpassing the A1 highway. It was a three span bridge with a main span of $30 \mathrm{~m}$ and two side spans of $14 \mathrm{~m}$ each. It was monitored for almost a year to collect different environmental parameters ${ }^{865}$ as well as acceleration measurements. The acceleration measurements were recorded for almost every hour and an automatic system identification system was in place to derive the modal parameters of the bridge. The stochastic subspace identification method was used to obtained the modal parameters of the structure [3]. The bridge was gradually damaged near the end of the monitoring period to create a benchmark structure for structural health monitoring. The damaged cases that the bridge was subjected with are presented in Table 2 and a detailed description of the cases can be found in Kramer et al. 36.

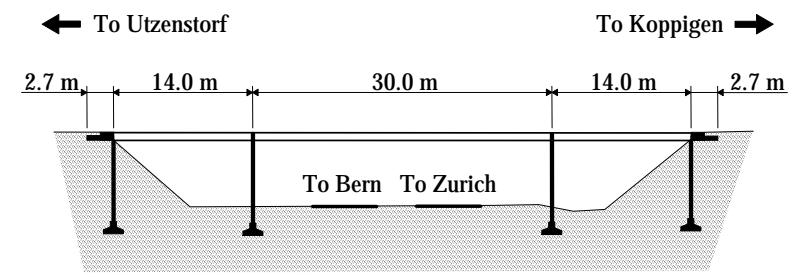

Figure 10: Z24 Bridge (Adapted from Peeters and De Roeck[3]).

Table 2: Description of the progressive damage cases applied to the Z24 Bridge (Adapted from Reynders et al. [37]).

\begin{tabular}{|c|c|}
\hline Test $\mathrm{N}$ 음 & Case description \\
\hline 1 & Reference state \\
\hline 2 & System installation for pier settlement \\
\hline 3 & $20 \mathrm{~mm}$ settlement of pier \\
\hline 4 & $40 \mathrm{~mm}$ settlement of pier \\
\hline 5 & $80 \mathrm{~mm}$ settlement of pier \\
\hline 6 & $95 \mathrm{~mm}$ settlement of pier \\
\hline 7 & Foundation tilt \\
\hline 8 & New reference state \\
\hline 9 & $12 \mathrm{~m}^{2}$ chipping of concrete \\
\hline 10 & $24 \mathrm{~m}^{2}$ chipping of concrete \\
\hline 11 & Landslide \\
\hline 12 & Concrete hinges failure \\
\hline 13 & 2 anchor heads failure \\
\hline 14 & 4 anchor heads failure \\
\hline 15 & Rupture of 2 tendons out of 16 \\
\hline 16 & Rupture of 4 tendons out of 16 \\
\hline 17 & Rupture of 6 tendons out of 16 \\
\hline
\end{tabular}

The first four natural frequencies of the structure along with several environmental and operational parameters that were monitored were made available to researchers for structural health monitoring. For damage detection, the first four natural frequencies of the structure are analysed using the method developed in this paper. Plots of the first four natural frequencies of the bridge versus the ambient temperature conditions are given in Fig. 11. It can be seen from the figure that the undamaged observations (black dots) have a nonlinear relationship with the ambient temperature conditions. Peeters and De Roeck 3] suggested that the nonlinear relationship was attributed to the asphalt layer on the bridge, which at temperatures below zero degrees, contributed to the stiffness of the structure, while at warmer temperatures, it had less influence. Therefore, the polynomial regression analysis is adopted 
in this paper to take into account this nonlinear effects. of vibration is more affected by the presence of damage (damaged observations given by red dots) than the other modes. Therefore, this difference can be analysed using $9_{930}$ the method developed in this paper to detect damage.

To apply the damage detection method proposed in this paper, regression models representing the undamaged state of the structure need to be created first. The first 3000 undamaged observations are used to create the re-935 gression models, and these observations are used because

880 they cover the whole range of temperature conditions that the structure encountered. As mentioned previously, it is ideal to use measurements obtained from a wide range of environmental and operational conditions to construct the 940 regression models. Another 200 undamaged observations

${ }_{885}$ are used to validate the models created. The rest of the undamaged cases and all the damaged cases are used for testing.

Similar to the beam structure case study, six regres-945 sion models are created using the first, second, third and

890 fourth natural frequencies as the independent and dependent variables. The difference in R-Squared between two consecutive polynomial orders is also used to determine the polynomial order of the regression models and the mod-950 els are validated by analysing the 200 undamaged observa-

895 tions. The percentage change in R-Squared for the first six degrees of polynomial for all pairs of natural frequencies are presented in Table 3. The polynomial order adopted for each pair of natural frequencies is also highlighted us-955 ing the symbol '*' in Table 3 . For example, a quadratic

900 polynomial is adopted for the model representing the relationship between the first and second natural frequencies.

After the regression models have been constructed, the natural frequencies of the dependent variable of new ob-960 servations are predicted using the values of natural frequencies of the independent variable obtained from field measurements. Then, the difference between the predicted value of the dependent variable and the value of the dependent variable obtained from field measurements is used as965 a deviation index and is analysed using an outlier analy-

${ }_{910}$ sis. The results of the outlier analysis are presented in Fig. 12 for all the undamaged and damaged cases analysed. It should be noted that prior to analysing the undamaged and damaged cases used for testing, the models are vali-970 dated using the 200 undamaged observations.

In Fig. 12 the dots are the observations being analysed while the two dotted horizontal lines are the control limits defining the normal range of conditions. The vertical line in each plot separates the undamaged and damaged cases.975 From Fig. 12, it can be seen that most of the undamaged cases are within the control limits in all six plots (six pairs of natural frequencies model), indicating that no damage is present in the bridge structure. There is only a fluctuation in the deviation index which is attributed to the 980 changing environmental and operational conditions (e.g.
For the damaged cases, when the second natural frequency is not included in the pair of frequencies (Figs. 12 (b, c and e)), almost all of the observations are within the control limits. There is only a small step change in deviation index from the undamaged cases, and a fluctuation in deviation index due to the changing environmental and operational conditions, similar to the undamaged cases. The step change exists because, the damaged cases are not exactly in the center of the undamaged cases database. They are in the space where the undamaged observations have high noise levels and large data processing errors (around the main cluster of undamaged observations) as can be seen in Figs. 11(a, c and d). When the second natural frequency is included in the pair of frequencies (Figs. 12(a, d and f)), most of the damaged cases are outside the control limits, indicating the presence of damage. The second natural frequency was strongly affected by the presence of damage while the other three frequencies were less affected. This creates a change in relationship between the pair of frequencies from the undamaged condition, hence the damaged observations do not follow the regression model (a new relationship is created between the pair of frequencies due to the presence of damage, and is different to the relationship of the undamaged condition). The deviation index also increases with the increase in damage extent. Therefore, in addition of alerting damage, the method proposed in this paper can also give the change in damaged scenarios.

For damage detection, the results obtained from each regression model are grouped together and the damage alert is raised when the monitored observation is outside the control limits in at least one of the model. The successful rates of alerting healthy condition for the undamaged cases and alerting damage for the damaged cases are $95.6 \%$ and $95.9 \%$, respectively. Some of the undamaged cases are alerted as damage and this may be attributed to the effects of high noise level and large data processing errors while extracting the natural frequencies. Soo Lon Wah et al. 31 mentioned that a database of natural frequencies usually contains some measurements that are subjected to high noise levels and large data processing errors. These measurements are usually not in the range of the normal conditions. Although some of the damaged cases are not alerted as damage, the performance of the method developed in this paper for damage detection is deemed satisfactory.

The damage detection method developed in this paper analyses the difference between the most affected mode of vibration and the least affected mode of vibration due to the effects of damage, to raise the damage alert. For example, for the beam structure model, the first, second and fourth natural frequencies were affected by damage while the third natural frequency was relatively unaffected. By analysing the difference between the third natural frequency and the other frequencies, damage detection can be achieved as can be seen in Fig. 8. Similarly, for the Z24 Bridge, when the most affected natural frequency (the 
(a)

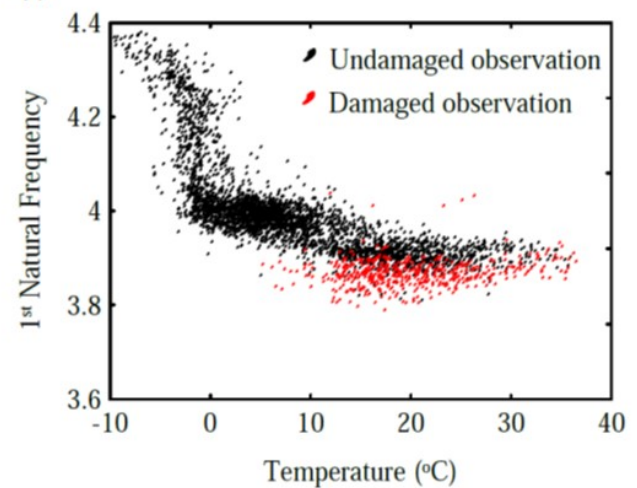

(c)

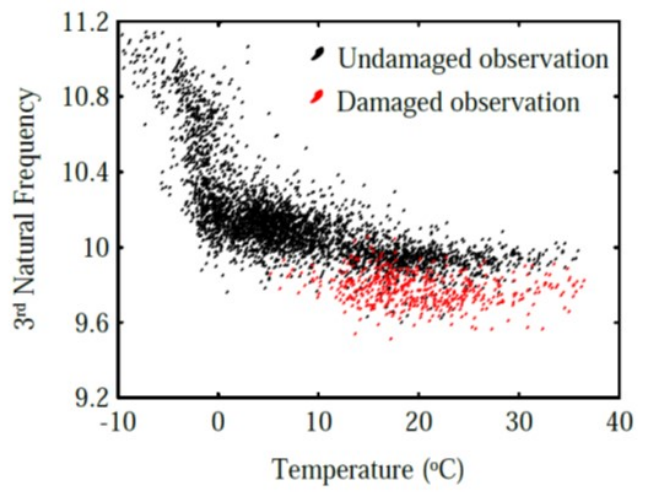

(b)

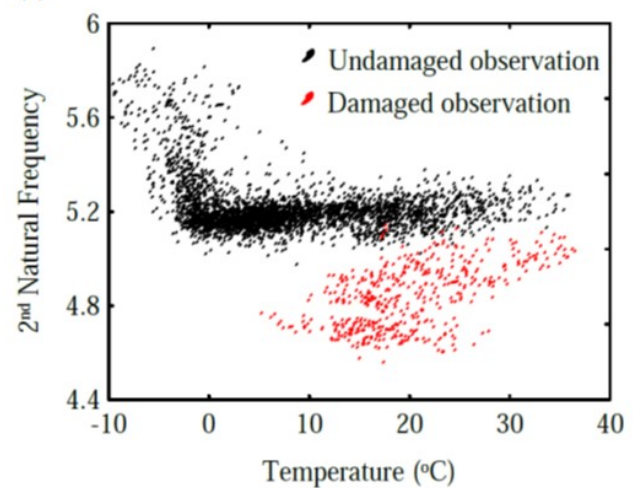

(d)

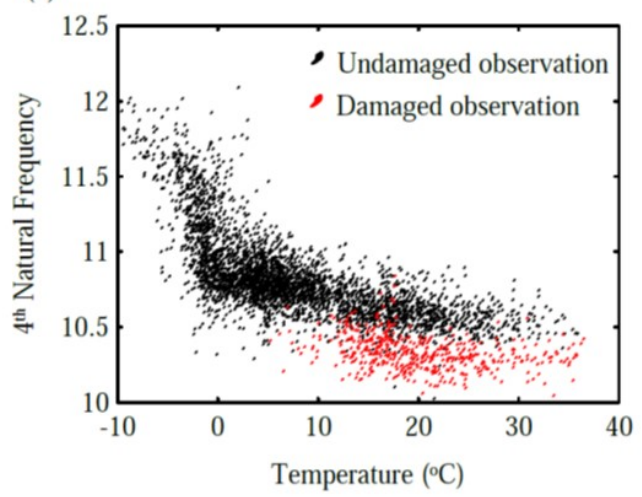

Figure 11: Plot of temperature versus (a) $1^{\text {st }}$ natural frequency, (b) $2^{\text {nd }}$ natural frequency, (c) $3^{\text {rd }}$ natural frequency and (d) $4^{\text {th }}$ natural frequency of the Z24 Bridge.

Table 3: Percentage change in R-Squared from previous polynomial order. Chosen polynomial order highlighted with the symbol ' $*$ '.

\begin{tabular}{ccccccc}
\hline Polynomial order & 1NF, 2NF & 1NF, 3NF & 1NF, 4NF & 3NF, 2NF & $3 N F, 4 N F$ & $4 N F, 2 N F$ \\
\hline 1 & - & $-*$ & $-*$ & - & $-*$ & - \\
2 & $35^{*}$ & 0 & 0 & $36^{*}$ & 1 & $43^{*}$ \\
3 & 0 & 0 & 0 & 0 & 0 & 0 \\
4 & 0 & 0 & 0 & 0 & 0 & 0 \\
5 & 0 & 0 & 0 & 0 & 0 & 0 \\
6 & 0 & 0 & 0 & 0 & 0 & 0 \\
\hline
\end{tabular}

second natural frequency) was compared with the least affected one (the first, third and fourth modes of vibrationooo were less affected), the presence of damage can be seen. For damage detection, it is important to construct a model between each pair of natural frequencies so that the most affected and the least affected modes of vibration can be compared with each other for any damaged scenarios. ${ }_{1005}$

The traditional regression damage detection method is also applied to the data of the Z24 Bridge for comparison. The ambient temperature condition is used as the independent variable while all four natural frequencies are used as the dependent variables. The first 3000 undam 010 aged observations are used to create the regression models and another 200 undamaged observations are used to validate the models. The rest of the undamaged cases and all the damaged cases are used for testing. The success- ful rates for the undamaged and damaged cases using the traditional regression damage detection method are 98.9 $\%$ and $83.5 \%$, respectively. Some of the damaged cases were not alerted as damage because, as can be seen in Fig. 11. most of the damaged cases cluster together with the undamaged cases in the space containing the observations with high noise levels and large data processing errors. Some of the damaged cases are also in the main cluster of the undamaged cases in the first, third and fourth natural frequency - temperature conditions plots (Figs. 11(a, c and $\mathrm{d})$ ).

From the results obtained using both damage detection methods, it can be seen that better performances are obtained for the damaged cases using the method developed in this paper with an improvement of $12 \%$. For the undamaged cases, slightly better result is obtained us- 
(a)

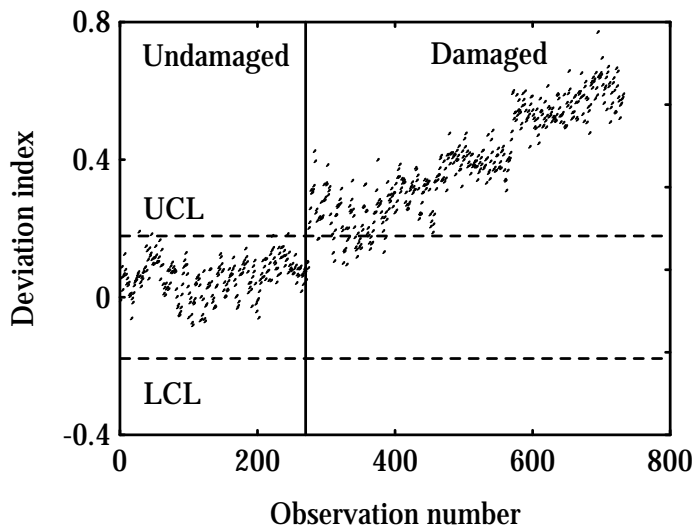

Independent variable: 1NF, D ependent variable: $2 \mathrm{NF}$

(c)

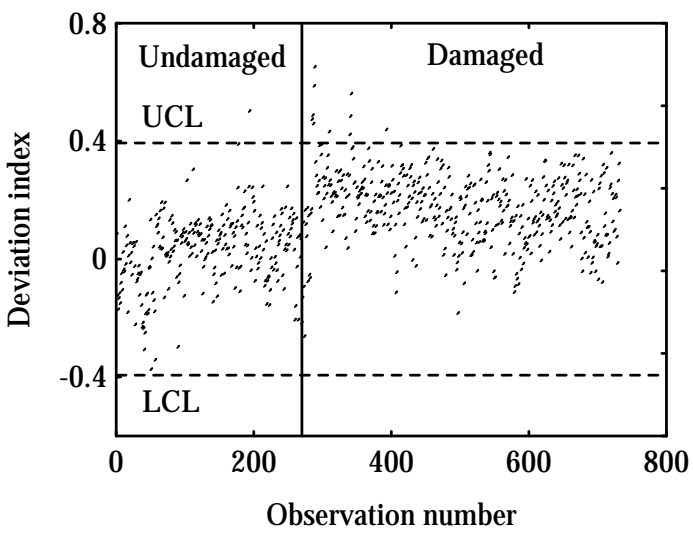

Independent variable: 1NF, Dependent variable: 4NF

(e)

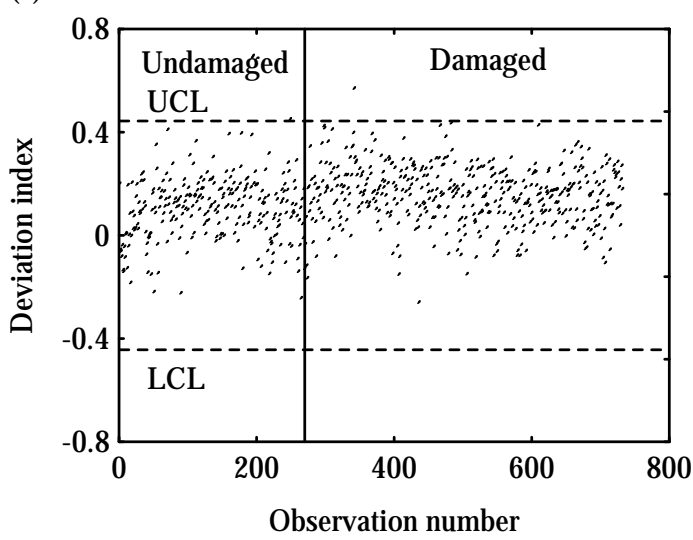

Independent variable: 3NF, Dependent variable: 4NF (b)

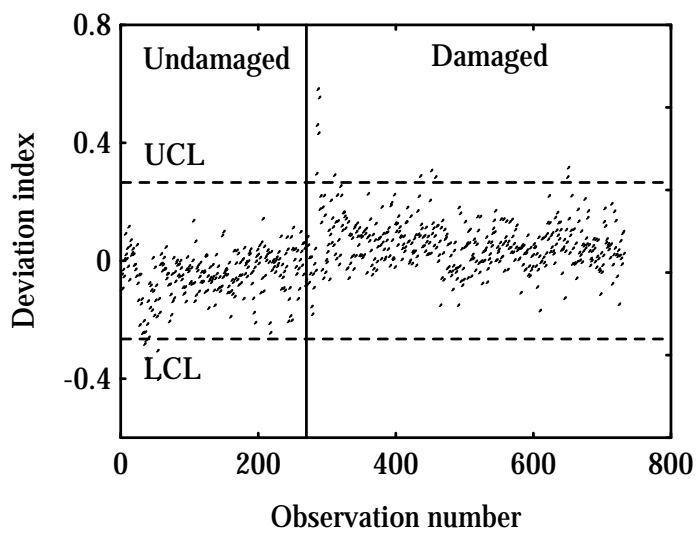

Independent variable: $1 \mathrm{NF}$, Dependent variable: 3NF

(d)

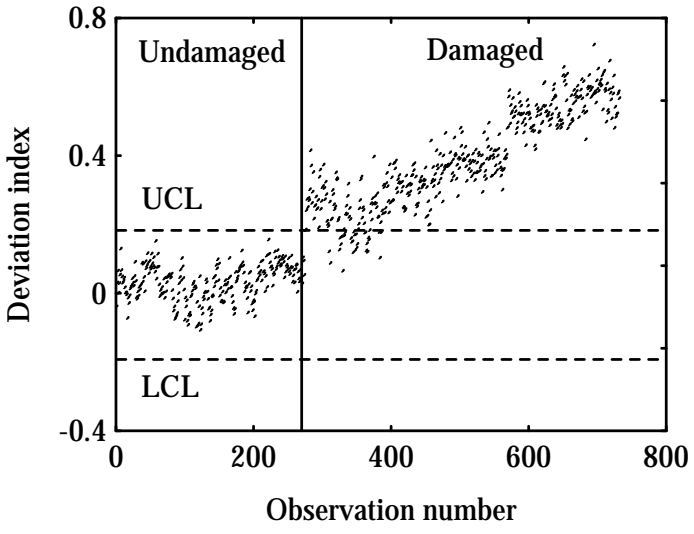

Independent variable: 3NF, Dependent variable: $2 \mathrm{~N} \mathrm{~F}$

(f)

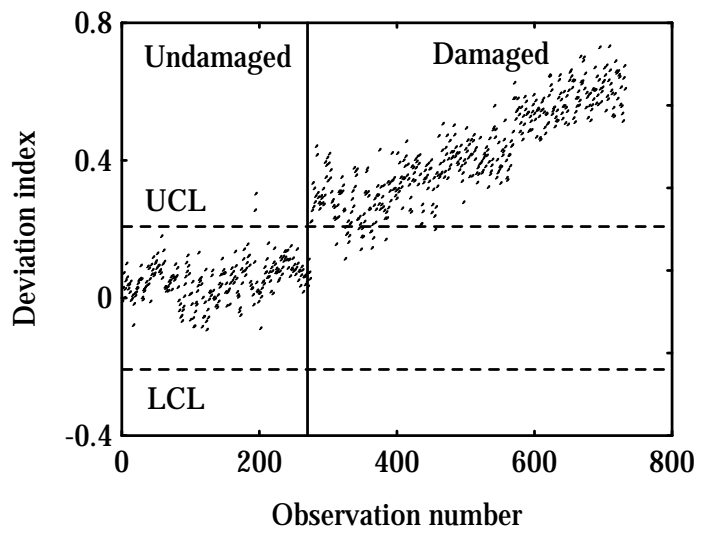

Independent variable: 4NF, Dependent variable: $2 \mathrm{NF}$

Figure 12: Results of outlier analysis of the Z24 Bridge using the method developed in this paper. *NF represents natural frequency.

ing the traditional regression damage detection method. As mentioned previously, the undamaged cases that are alerted as damage may be due to high noise levels and large errors while extracting the frequencies. To reduce these false alerts, it is proposed to introduce a threshold 025 vations is outside the control limits that the damage alert will be raised. For example, in this paper it is proposed to use 2 and 3 consecutive observations as the threshold. This means that only when 2 (or 3 depending on which one was chosen) consecutive observations are outside the control limits that the damage alert is raised. The results of 
applying these two thresholds with the damage detection method developed in this paper and with the traditional regression analysis method are given in Table 4.

From Table 4, it can be seen that better results are obtained (using both methods) for the undamaged cases when the thresholds are applied. When the 3 consecutive observations is adopted as the threshold, $100 \%$ successful rate is obtained for the undamaged cases. The observations with high noise levels and large data processing errors have been taken into account using the thresholds. This is because, observations with high noise levels and large errors are usually not continuous. Therefore, establishing the consecutive observations threshold, the false alerts are reduced. For the damaged cases, however, as the num ${ }_{1095}$ ber of consecutive observation increases for the threshold, the successful rate decreases for both methods. This is because, observations that are outside the control limits are considered as not damaged when they are followed by or they follow an observation that is within the control limits. A compromise need to be found between alerting damage for the damaged cases and not raising the damage alert for the undamaged cases subjected to high noise levels and data processing errors.

Although the successful rate decreases for both methods for the damaged cases, the damage detection method developed in this paper has better performances than the traditional regression analysis method. In addition of having better performances, the developed method eliminates the limitations of the damage detection methods proposed $_{110}$ in the literature mentioned previously in the 'Introduction' section. The environmental and operational parameters were not needed to apply the damage detection method. A single data set containing all the vibration properties was not required to be analysed simultaneously to extract new features that are sensitive to damage but less sensitive to the changing environments. The method also does not require a regression target, which is difficult to determine, to be chosen. Moreover, the nonlinear effects from the 1065 changing environmental and operational conditions can be taken into account using the damage detection method developed in this paper.

In the method developed in this paper, the natural frequencies of structures were used as both the independent120 and dependent variables, while for the traditional regression damage detection method, the temperature condition was used as the independent variable and the frequencies were the dependent variables. The temperature condition was used as the independent variable because although both structures analysed were subjected to multiple effects ${ }^{125}$ (e.g. variations in temperature and mass for the beam and the likes of temperature and wind loading for the Z24 Bridge), it was assumed that temperature was the main environmental effect affecting the structures. However, for ${ }^{1130}$ other civil engineering structures, a combination of multiple main environmental and operational effects can also affect the structures. Hence, more work need to be carried out to analyse structures where several main environmen- tal and operational conditions are present.

\section{Conclusion}

A damage detection method is developed in this paper using the regression analysis approach with the natural frequencies of the structures used as the independent and dependent variables. The method is applied to a beam structure and the Z24 Bridge, in Switzerland. The traditional regression damage detection method is also applied to the two case studies for comparison. Although both methods can detect the presence of damage, better results are obtained using the method developed in this paper. Analysing the relationships among the natural frequencies and comparing the least affected to the most affected modes of vibration, allow the method to be more sensitive to damage. In addition of having better performances, the method developed in this paper does not require the environmental and operational parameters for analysis, which reduces the risk of affecting the performance of the damage detection method. Moreover, all the natural frequencies were not processed simultaneously in a single data set using multivariate statistical tools, which reduces false alerts. The nonlinear effects from the changing environments can also be taken into account using the developed method. The use of a threshold is also introduced in this paper to reduce the occurrence of false alerts due to high noise levels and large data processing errors. The successful rates for the undamaged cases increases when the threshold is used.

\section{Acknowledgment}

The authors would like to acknowledge Prof. Guido De Roeck and Prof. Edwin Reynders for providing the data of the Z24 Bridge. This work is supported by Ningbo Science and Technology Bureau under Commonweal Research Programme with project code 2019C50017 and a research grant with project code A0060 from Ningbo Nottingham New Material Institute.

\section{References}

\section{References}

[1] H. Sohn, Effects of environmental and operational variability on structural health monitoring, Philos Trans Royal Soc A 365 (1851) (2007) 539-560.

[2] W. Soo Lon Wah, Y.-T. Chen, A new approach toward damage localization and quantification of structures under changing temperature condition, J Low Freq Noise V A (2018) 1-16.

[3] B. Peeters, G. De Roeck, One-year monitoring of the z24-bridge: environmental effects versus damage events, Earthq Eng Struct Dyn 30 (2) (2001) 149-171.

[4] S. Desjardins, N. Londono, D. Lau, H. Khoo, Real-time data processing, analysis and visualization for structural monitoring of the confederation bridge, Adv Struct Eng 9 (1) (2006) 141157. 
Table 4: Successful rates (\%) of the damage detection methods applied to the Z24 Bridge. * 'Proposed' means the method proposed in this paper and 'Traditional' means the traditional regression analysis method.

\begin{tabular}{ccccccc}
\hline & \multicolumn{2}{c}{ No consecutive } & \multicolumn{2}{c}{ 2 consecutive } & \multicolumn{2}{c}{3 consecutive } \\
\hline Cases & Proposed & Traditional & Proposed & Traditional & Proposed & Traditional \\
\hline Undamaged & 95.6 & 98.9 & 99.3 & 99.3 & 100 & 100 \\
Damaged & 95.9 & 83.5 & 94.4 & 77.5 & 92.9 & 74.7 \\
\hline
\end{tabular}

[5] P. Moser, B. Moaveni, Environmental effects on the identified195 natural frequencies of the dowling hall footbridge, Mech Syst Signal Pr 25 (7) (2011) 2336-2357.

[6] C. Jin, J. Li, S. Jang, X. Sun, R. Christenson, Structural damage detection for in-service highway bridge under operational and environmental variability, in: Sensors and Smart Struc $¥ 200$ tures Technologies for Civil, Mechanical, and Aerospace Systems 2015, International Society for Optics and Photonics, 2015.

[7] P. Salcher, H. Pradlwarter, C. Adam, Reliability assessment of railway bridges subjected to high-speed trains considering the effects of seasonal temperature changes, Eng Struct 126 (2016 205 $_{205}$ $712-724$

[8] J.-X. Mao, H. Wang, D.-M. Feng, T.-Y. Tao, W.-Z. Zheng, Investigation of dynamic properties of long-span cable-stayed bridges based on one-year monitoring data under normal operating condition, Struct Control Hlth 25 (5).

[9] W. Soo Lon Wah, J. S. Owen, Y.-T. Chen, A. Elamin, G. W. Roberts, Damage detection of a cable-stayed bridge subjected to changing environmental and operational conditions, in: Proceedings of the 7th World Conference on Structural Control and Monitoring, Qingdao, China, 22-25 July 2018.

10] Q. Zhang, L. Fan, W. Yuan, Traffic-induced variability in dynamic properties of cable-stayed bridge, Earthq Eng Struct Dyn 31 (11) (2002) 2015-2021.

[11] E. Cross, K. Koo, J. Brownjohn, K. Worden, Long-term monitoring and data analysis of the tamar bridge, Mech Syst Signał 220 Pr 35 (1-2) (2013) 16-34.

[12] X. Meng, D. T. Nguyen, Y. Xie, J. S. Owen, P. Psimoulis, S. Ince, Q. Chen, J. Ye, P. Bhatia, Design and implementation of a new system for large bridge monitoring geoshm, Sensors 18 (3) (2018) 775-797.

[13] D. M. Siringoringo, Y. Fujino, System identification of suspension bridge from ambient vibration response, Eng Struct $30(2)$ (2008) 462-477.

[14] Y. Xia, H. Hao, G. Zanardo, A. Deeks, Long term vibration monitoring of an rc slab: temperature and humidity effect, Eng $_{230}$ Struct 28 (3) (2006) 441-452.

[15] O. Büyüköztürk, T.-Y. Yu, Structural health monitoring and seismic impact assessment, in: Proceedings of the 5th National Conference on Earthquake Engineering, 2003.

[16] C. Fritzen, G. Mengelkamp, A. Guemes, Elimination of tem ${ }_{1235}$ perature effects on damage detection within a smart structure concept, in: Proceedings of the 4th International Workshop on Structural Health Monitoring, Stanford, CA, 15-17 September 2003, pp. 1530-1538.

[17] N. Dervilis, K. Worden, E. Cross, On robust regression analysi\$240 as a means of exploring environmental and operational conditions for shm data, J Sound Vib 347 (2015) 279-296.

[18] K. Worden, H. Sohn, C. Farrar, Novelty detection in a changing environment: regression and interpolation approaches, J Sound Vib 258 (4) (2002) 741-761.

[19] B. Peeters, J. Maeck, G. De Roeck, Dynamic monitoring of the z24-bridge: separating temperature effects from damage, in: Proceedings of the European COST F3 Conference on System Identification and Structural Health Monitoring, Madrid, Spain, June 2000, pp. 377-386.

[20] Y.-Q. Ni, K. Fan, G. Zheng, T. H. Chan, J. M. Ko, Automatic modal identification of cable-supported bridges instrumented with a long-term monitoring system, in: Smart Structures and Materials 2003: Smart Systems and Nondestructive Evaluation for Civil Infrastructures, San Diego, CA, March 2003, pp. 329339.

[21] C. Gentile, M. Guidobaldi, A. Saisi, One-year dynamic monitoring of a historic tower: damage detection under changing environment, Meccanica 51 (11) (2016) 2873-2889.

[22] K. Worden, E. Cross, On switching response surface models, with applications to the structural health monitoring of bridges, Mech Syst Signal Pr 98 (2018) 139-156.

[23] A.-M. Yan, G. Kerschen, P. De Boe, J.-C. Golinval, Structural damage diagnosis under varying environmental conditions-part i: a linear analysis, Mech Syst Signal Pr 19 (4) (2005) 847-864.

24] J. M. Ko, K. K. Chak, J. Y. Wang, Y.-Q. Ni, T. H. Chan, Formulation of an uncertainty model relating modal parameters and environmental factors by using long-term monitoring data, in: Proceedings of Smart Structures and Materials 2003: Smart Systems and Nondestructive Evaluation for Civil Infrastructures, San Diego, CA, 2-6 March 2003, pp. 298-307.

[25] E. J. Cross, K. Worden, Q. Chen, Cointegration: a novel approach for the removal of environmental trends in structural health monitoring data, P Roy Soc Lond A Mat 467 (2133) (2011) 2712-2732.

[26] G. Comanducci, F. Magalhães, F. Ubertini, Á. Cunha, On vibration-based damage detection by multivariate statistical techniques: Application to a long-span arch bridge, Struct Health Monit 15 (5) (2016) 505-524.

[27] W. Soo Lon Wah, Y.-T. Chen, G. W. Roberts, A. Elamin, Separating damage from environmental effects affecting civil structures for near real-time damage detection, Struct Health Monit 17 (4) (2017) 850-868.

[28] W. Soo Lon Wah, Y.-T. Chen, G. W. Roberts, A. Elamin, Damage detection of structures subject to nonlinear effects of changing environmental conditions, in: Proceedings of the 6th Asia-Pacific Workshop on Structural Health Monitoring, Hobart, Australia, 7-9 December 2016, 2017, pp. 248-255.

[29] W. Soo Lon Wah, Y.-T. Chen, A. Elamin, G. W. Roberts, Damage detection under temperature conditions using pca-an application to the z24 bridge, Proceedings of the Institution of Civil Engineers-Structures and Buildings (2020) 1-13.

[30] H. Shi, K. Worden, E. Cross, A nonlinear cointegration approach with applications to structural health monitoring, in: Journal of Physics: Conference Series, Vol. 744, IOP Publishing, 2016, p. 012025.

[31] W. Soo Lon Wah, J. S. Owen, Y.-T. Chen, A. Elamin, G. W. Roberts, Removal of masking effect for damage detection of structures, Eng Struct 183 (2019) 646-661.

[32] J. O. Rawlings, S. G. Pantula, D. A. Dickey, Applied regression analysis: a research tool, Springer Science \& Business Media, 2001.

[33] Y. Ding, A. Li, Temperature-induced variations of measured modal frequencies of steel box girder for a long-span suspension bridge, Int J Steel Struct 11 (2) (2011) 145-155.

[34] N. J. Salkind, Encyclopedia of research design, Vol. 1, Sage, 2010

[35] J. Kullaa, Distinguishing between sensor fault, structural damage, and environmental or operational effects in structural health monitoring, Mech Syst Signal Pr 25 (8) (2011) 29762989.

[36] C. Krämer, C. De Smet, G. De Roeck, Z24 bridge damage detection tests, in: Proceedings of the 17th International Modal 
Analysis Conference, Kissimmee, FL, 8-11 February 1999, pp. 1023-1029.

[37] E. Reynders, G. Wursten, G. De Roeck, Output-only structural health monitoring in changing environmental conditions by means of nonlinear system identification, Struct Health Monit 13 (1) (2014) 82-93. 